\title{
Adler-Bardeen theorem and manifest anomaly cancellation to all orders in gauge theories
}

\author{
Damiano Anselmi ${ }^{\mathrm{a}}$ \\ Dipartimento di Fisica “Enrico Fermi”, Università di Pisa, and INFN, Sezione di Pisa, Largo B. Pontecorvo 3, 56127 Pisa, Italy
}

Received: 30 July 2014 / Accepted: 15 September 2014 / Published online: 7 October 2014

(C) The Author(s) 2014. This article is published with open access at Springerlink.com

\begin{abstract}
We reconsider the Adler-Bardeen theorem for the cancellation of gauge anomalies to all orders, when they vanish at one loop. Using the Batalin-Vilkovisky formalism and combining the dimensional-regularization technique with the higher-derivative gauge invariant regularization, we prove the theorem in the most general perturbatively unitary renormalizable gauge theories coupled to matter in four dimensions, and we identify the subtraction scheme where anomaly cancellation to all orders is manifest, namely no subtractions of finite local counterterms are required from two loops onwards. Our approach is based on an order-byorder analysis of renormalization, and, differently from most derivations existing in the literature, does not make use of arguments based on the properties of the renormalization group. As a consequence, the proof we give also applies to conformal field theories and finite theories.
\end{abstract}

\section{Introduction}

The Adler-Bardeen theorem [1,2] is a crucial property of quantum field theory, and one of the few tools to derive exact results. In the literature various statements go under the name of "Adler-Bardeen theorem". They apply to different situations. The original statement by Adler and Bardeen says that (I) the Adler-Bell-Jackiw axial anomaly [3,4] is one-loop exact. The second statement, which is the one we are going to study here, says that (II) (there exists a subtraction scheme where) gauge anomalies vanish to all orders, if they vanish at one loop. Statement II is important to justify the cancellation of gauge anomalies to all orders in the standard model. A third statement concerns the one-loop exactness of anomalies associated with external fields.

Statement I is expressed by a well-known operator identity for the divergence of the axial current. By means of a diagrammatic analysis, Adler and Bardeen were able to provide

a e-mail: damiano.anselmi@df.unipi.it the subtraction scheme where that identity is manifestly oneloop exact in QED [1]. They emphasized that higher-order corrections vanish, unless they contain the one-loop triangle diagram as a subdiagram. Thus stated, statement I intuitively implies statement II. However, the original proof of Adler and Bardeen applies only to QED.

Other approaches to the problem have appeared, since the paper by Adler and Bardeen, in Abelian and nonAbelian gauge theories. For a review, see for example [2]. Statement I can be proved using arguments based on the properties of the renormalization group [5-7], regularization independent algebraic techniques [8], or an algebraic/geometric derivation [9] based on the WessZumino consistency conditions [10] and the quantization of the Wess-Zumino-Witten action. Statement II can also be proved using renormalization-group (RG) arguments, with the dimensional regularization [11] or regularizationindependent approaches [12].

More recently, statement II was proved by the author of this paper in standard model extensions with highenergy Lorentz violation [13], which are renormalizable by "weighted power counting" [14]. The approach of [13] is closer to the original approach by Adler and Bardeen, in the sense that it does not make use of RG arguments, algebraic methods or geometric shortcuts, it naturally provides the subtraction scheme where the all-order cancellation is manifest, and it is basically a diagrammatic analysis, although instead of dealing directly with diagrams, it uses the BatalinVilkovisky formalism [15-17] to manage relations among diagrams in a compact and efficient way.

In the present paper we prove statement II in the most general perturbatively unitary, renormalizable gauge theories coupled to matter, and elaborate further along the guidelines of Ref. [13]. We upgrade the approach of [13] in a number of directions, emphasize properties that were not apparent at that time, and expand the arguments that were presented concisely. We also gain a certain clarity by dropping the Lorentz 
violation. A side purpose of this investigation is to develop new techniques and tools to prove all-order theorems in quantum field theory with a smaller effort.

Our results make progress in several directions. To our knowledge, if we exclude Ref. [13] and this paper, statement II has been proved beyond QED only making use of arguments based on the renormalization group. However, RG arguments do not provide the subtraction scheme where the all-order cancellation is manifest, and they are not sufficiently general. For example, they are powerless when the beta functions identically vanish, so they exclude conformal field theories and finite theories, where, however, the AdlerBardeen theorem does hold. Actually, RG techniques fail even when the first coefficients of the beta functions vanish $[11,12]$. Our approach does not suffer from these limitations. Another reason to avoid shortcuts is that in the past the Adler-Bardeen theorem caused some confusion in the literature, therefore new proofs, and even more generalizations, should be as transparent as possible. In this paper we pay attention to all details.

The all-order cancellation of gauge anomalies is a property that depends on the scheme, but the existence of a good scheme is not evident. Knowing the scheme where the cancellation is manifest is very convenient from the practical point of view, because it saves the effort of subtracting ad hoc finite local counterterms at each step of the perturbative expansion. For example, using the dimensional regularization and the minimal subtraction scheme the cancellation of two-loop and higher-order corrections to gauge anomalies in the standard model is not manifest, and finite local counterterms must be subtracted every time.

To find the right subtraction scheme we need to define a clever regularization technique. It turns out that using the Batalin-Vilkovisky formalism and combining the dimensional regularization with the gauge invariant higherderivative regularization, the subtraction scheme where the Adler-Bardeen theorem is manifest emerges quite naturally [13].

It is well known that, in general, gauge invariant higherderivative regularizations do not regularize completely, because some one-loop diagrams can remain divergent. From our viewpoint, this is not a weakness, because it allows us to separate the sources of potential anomalies from everything else. We just have to use a second regulator, the dimensional one, to deal with the few surviving divergent diagrams.

The regularization we are going to use introduces two cutoffs: $\varepsilon=4-D$, where $D$ is the continued complex dimension, and an energy scale $\Lambda$ for the higher-derivative regularizing terms. The regularized action must be gauge invariant in $D=4$, to ensure that the higher-derivative regulator has the minimum impact on gauge anomalies. The physical limit is defined letting $\varepsilon$ tend to 0 and $\Lambda$ to $\infty$. When we have two or more cutoffs, physical quantities do not depend on the order in which we remove them. More precisely, exchanging the order of the limits $\varepsilon \rightarrow 0$ and $\Lambda \rightarrow \infty$ is equivalent to change the subtraction scheme. That kind of scheme change is, however, crucial for our arguments.

Consider first the limit $\Lambda \rightarrow \infty$ followed by $\varepsilon \rightarrow 0$. When $D \neq 4$ the limit $\Lambda \rightarrow \infty$ is regular in every diagram and gives back the dimensionally regularized theory: no $\Lambda$ divergences appear, but just poles in $\varepsilon$. In this framework there are no known subtraction schemes where the AdlerBardeen theorem holds manifestly.

Now, consider the limit $\varepsilon \rightarrow 0$ followed by $\Lambda \rightarrow \infty$. At fixed $\Lambda$ we have a higher-derivative theory. If properly organized, that theory is superrenormalizable and contains just a few (one-loop) divergent diagrams, which are poles in $\varepsilon$ and may be removed by redefining some parameters. At a second stage, we study the limit $\Lambda \rightarrow \infty$, where $\Lambda$ divergences appear and are removed by redefining parameters and making canonical transformations. We call the regularization technique defined this way dimensional/higherderivative (DHD) regularization.

Intuitively, if gauge anomalies are trivial at one loop, there should be no further problems at higher orders, because the higher-derivative regularization is manifestly gauge invariant. Thus, we expect that the DHD regularization provides the framework where the Adler-Bardeen theorem is manifest. However, it is not entirely obvious that the two regularization techniques can be merged to achieve the goal we want. Among the other things, $\varepsilon$ evanescent terms are around all the time and the $\mathcal{O}\left(1 / \Lambda^{n}\right)$ regularizing terms can simplify power-like $\Lambda$ divergences, causing troubles. Nevertheless, with some effort and a nontrivial amount of work we can prove that all difficulties can be properly dealt with.

Summarizing, the statement we prove in this paper is

Theorem In renormalizable perturbatively unitary gauge theories coupled to matter, there exists a subtraction scheme where gauge anomalies manifestly cancel to all orders, if they are trivial at one loop.

Once we have this result, we know that no matter what scheme we use, it is always possible to find ad hoc finite local counterterms that ensure the cancellation of gauge anomalies to all orders. Then we are free to use the more common minimal subtraction scheme and the pure dimensional regularization technique.

The paper is organized as follows. In Sects. 2-7 we prove the theorem in non-Abelian Yang-Mills theory coupled to left-handed chiral fermions. This model is sufficiently general to illustrate the key points of the proof, as well as the main arguments and tools, but relatively simple to free the derivation from unnecessary complications. At the end of the paper, in Sect. 8, we show how to include the missing fields, namely right-handed fermions, scalars, and photons, and cover the most general perturbatively unitary renormal- 
izable gauge theory coupled to matter. Section 9 contains our conclusions. In Appendix A we recall the calculation of gauge anomalies in chiral theories. In Appendix B we recall the proof of a useful formula.

The proof for Yang-Mills theory coupled to chiral fermions is organized as follows. In Sects. 2 and 3 we formulate the dimensional and DHD regularization techniques. In Sects. 4-6 we prove the Adler-Bardeen theorem in the higher-derivative theory, studying the limit $\varepsilon \rightarrow 0$ at $\Lambda$ fixed. Precisely, in Sect. 4 we work out the renormalization, in Sect. 5 we study the one-loop anomalies and in Sect. 6 we prove the anomaly cancellation to all orders. In Sect. 7 we take the limit $\Lambda \rightarrow \infty$ and conclude the proof of the Adler-Bardeen theorem for the final theory.

\section{Dimensional regularization of chiral Yang-Mills theory}

We first prove the Adler-Bardeen theorem in detail in fourdimensional non-Abelian Yang-Mills theory coupled to lefthanded chiral fermions. This model offers a sufficiently general arena to illustrate the key arguments and tools of our approach. At the same time, we make some clever choices to prepare the generalization (discussed in Sect. 8) to the most general perturbatively unitary gauge theories coupled to matter. To begin with, in this section we dimensionally regularize chiral gauge theories and point out a number of facts and properties that are normally not emphasized, but are rather important for the arguments of this paper.

Consider a gauge theory with gauge group $G$ and lefthanded chiral fermions $\psi_{L}^{I}$ in certain irreducible representations $R_{L}^{I}$ of $G$. If $G$ is the product of various simple groups $G_{i}$, we use indices $a, b, \ldots$ for $G$ and indices $a_{i}, b_{i}, \ldots$ for $G_{i}$. Denote the gauge coupling $g_{i}$ of each $G_{i}$ with $g r_{i}$, where $r_{i}$ are parameters of order one that we incorporate into the $G$ structure constants $f^{a b c}$ and the anti-Hermitian matrices $T^{a}$ associated with the representations of matter fields. We call $g$ the overall gauge coupling. We organize the matrices $T^{a}$ in block-diagonal form, where each block refers to a $\psi_{L}^{I}$ and its representation $R_{L}^{I}$. When we write $T^{a} \psi_{L}^{I}$ we understand that $T^{a}$ is replaced by the appropriate block. More fermions in the same irreducible representations may be present. With these conventions, the matrices $T^{a}$ still satisfy $\left[T^{a}, T^{b}\right]=f^{a b c} T^{c}$ and the classical action reads

$S_{c}=-\frac{1}{4} \sum_{i} \zeta_{i} \int F_{\mu \nu}^{a_{i}} F^{a_{i} \mu \nu}+\int \bar{\psi}_{L}^{I} l \not D \psi_{L}^{I}$,

where $F_{\mu \nu}^{a_{i}}=\partial_{\mu} A_{\nu}^{a_{i}}-\partial_{\nu} A_{\mu}^{a_{i}}+g_{i} f^{a_{i} b_{i} c_{i}} A_{\mu}^{b_{i}} A_{\nu}^{c_{i}}$ (no sum over this kind of index $i$ being understood, here and in the rest of the paper) is the $G_{i}$ field strength, $D_{\mu} \psi_{L}^{I}=\partial_{\mu} \psi_{L}^{I}+$ $g T^{a} A_{\mu}^{a} \psi_{L}^{I}$ is the fermion covariant derivative and $\iota$ is used for $\sqrt{-1}$ to avoid confusion with the index $i$. The parameters $\zeta_{i}$ could be normalized to 1 , but for future uses it is convenient to keep them free, because they are renormalized by poles in $\varepsilon$. Analogous parameters in front of the fermionic kinetic terms are not necessary.

To keep the presentation simple we make some simplifying assumptions that do not restrict the validity of our arguments. Specifically, we do not include right-handed fermions and scalar fields, and assume that the groups $G_{i}$ are nonAbelian, so there is no renormalization mixing among gauge fields, even when more copies of the same simple group are present. In Sect. 8 we explain how to relax these assumptions and cover the most general Abelian and non-Abelian perturbatively unitary renormalizable gauge theories coupled to matter.

Let us briefly recall the Batalin-Vilkovisky formalism for general gauge theories [15-17]. The classical fields $\phi=$ $\left\{A_{\mu}^{a}, \psi_{L}^{I}, \bar{\psi}_{L}^{I}\right\}$, together with the ghosts $C$, the antighosts $\bar{C}$ and the Lagrange multipliers $B$ for the gauge fixing are collected into the set of fields $\Phi^{\alpha}=\left\{A_{\mu}^{a}, C^{a}, \bar{C}^{a}, B^{a}, \psi_{L}^{I}, \bar{\psi}_{L}^{I}\right\}$. An external source $K_{\alpha}$ with opposite statistics is associated with each $\Phi^{\alpha}$, and coupled to the $\Phi^{\alpha}$ transformations $R^{\alpha}(\Phi, g)$. We have $K_{\alpha}=\left\{K^{\mu a}, K_{C}^{a}, K_{\bar{C}}^{a}, K_{B}^{a}, K_{\psi}^{I}, \bar{K}_{\psi}^{I}\right\}$. If $X$ and $Y$ are functionals of $\Phi$ and $K$ their antiparentheses are defined as

$(X, Y) \equiv \int\left(\frac{\delta_{r} X}{\delta \Phi^{\alpha}} \frac{\delta_{l} Y}{\delta K_{\alpha}}-\frac{\delta_{r} X}{\delta K_{\alpha}} \frac{\delta_{l} Y}{\delta \Phi^{\alpha}}\right)$,

where the integral is over spacetime points associated with repeated indices. The master equation $(S, S)=0$ must be solved with the "boundary condition" $S(\Phi, K)=S_{c}(\phi)$ at $C=\bar{C}=B=K=0$ in $D=4$, where $S_{c}(\phi)$ is the classical action (2.1). The solution $S(\Phi, K)$ is the action we start with to quantize the theory.

In the model we are considering the gauge algebra closes off shell, so there exists a variable frame where $S(\Phi, K)$ is linear in $K$. The non-gauge-fixed solution of the master equation is

$S_{\mathrm{ngf}}(\Phi, K)=S_{c}(\phi)+S_{K}$,

where the functional

$$
\begin{aligned}
S_{K}(\Phi, K)= & -\int R^{\alpha}(\Phi, g) K_{\alpha}=-\int\left(D_{\mu} C^{a}\right) K^{\mu a} \\
& +\frac{g}{2} \int f^{a b c} C^{b} C^{c} K_{C}^{a}-\int B^{a} K_{\bar{C}}^{a} \\
& +g \int\left(\bar{\psi}_{L}^{I} T^{a} C^{a} K_{\psi}^{I}+\bar{K}_{\psi}^{I} T^{a} C^{a} \psi_{L}^{I}\right)
\end{aligned}
$$

collects the symmetry transformations of the fields, $D_{\mu} C^{a}=$ $\partial_{\mu} C^{a}+g f^{a b c} A_{\mu}^{b} C^{c}$ being the covariant derivative of the ghosts. The gauge-fixed solution of the master equation reads 
$S_{\mathrm{gf}}(\Phi, K)=S_{\mathrm{ngf}}+\left(S_{K}, \Psi\right)=S_{c}(\phi)+\left(S_{K}, \Psi\right)+S_{K}$,

where $\Psi(\Phi)$ is the "gauge fermion", a functional of ghost number -1 that collects the gauge-fixing conditions. For convenience, we choose standard linear gauge-fixing conditions and write

$\Psi(\Phi)=\int \sum_{i} \bar{C}^{a_{i}}\left(\partial^{\mu} A_{\mu}^{a_{i}}+\frac{\xi_{i}}{2} B^{a_{i}}\right)$

where $\xi_{i}$ are gauge-fixing parameters.

The naïve $D$-dimensional continuation of the action (2.1) is not well regularized, because chiral fermions do not have good propagators. To overcome this difficulty, we proceed as follows. As usual, we split the $D$-dimensional spacetime manifold $\mathbb{R}^{D}$ into the product $\mathbb{R}^{4} \times \mathbb{R}^{-\varepsilon}$ of ordinary fourdimensional spacetime $\mathbb{R}^{4}$ times a residual $(-\varepsilon)$-dimensional evanescent space $\mathbb{R}^{-\varepsilon}$. Spacetime indices $\mu, v, \ldots$ of vectors and tensors are split into bar indices $\bar{\mu}, \bar{v}, \ldots$, which take the values $0,1,2,3$, and formal hat indices $\hat{\mu}, \hat{v}, \ldots$, which denote the $\mathbb{R}^{-\varepsilon}$ components. For example, the momenta $p^{\mu}$ are split into pairs $p^{\bar{\mu}}, p^{\hat{\mu}}$, or equivalently $\bar{p}^{\mu}, \hat{p}^{\mu}$. The flatspace metric $\eta_{\mu \nu}=\operatorname{diag}(1,-1, \ldots,-1)$ is split into $\eta_{\bar{\mu}} \bar{\nu}=$ $\operatorname{diag}(1,-1,-1,-1)$ and $\eta_{\hat{\mu} \hat{\nu}}=-\delta_{\hat{\mu} \hat{\nu}}$. When we contract evanescent components we use the metric $\eta_{\hat{\mu} \hat{v}}$, so for example $\hat{p}^{2}=p^{\hat{\mu}} \eta_{\hat{\mu} \hat{v}} p^{\hat{v}}$. We assume that the continued $\gamma$ matrices $\gamma^{\mu}$ satisfy the continued Dirac algebra $\left\{\gamma^{\mu}, \gamma^{\nu}\right\}=2 \eta^{\mu \nu}$. We define $\gamma_{5}=\imath \gamma^{0} \gamma^{1} \gamma^{2} \gamma^{3}, P_{L}=\left(1-\gamma_{5}\right) / 2, P_{R}=(1+$ $\left.\gamma_{5}\right) / 2$ and the charge-conjugation matrix $C=-l \gamma^{0} \gamma^{2}$ in the usual fashion. Full $\mathrm{SO}(1, D-1)$ invariance is lost in most expressions, replaced by $\mathrm{SO}(1,3) \times \mathrm{SO}(-\varepsilon)$ invariance.

The action (2.1) gives the fermion propagator $P_{L}(l / \bar{p}) P_{R}$, which involves only the four-dimensional components $\bar{p}^{\mu}$ of momenta. Therefore, it does not fall off in all directions of integration for $p \rightarrow \infty$. Applying the rules of the dimensional regularization, fermion loops integrate to zero. To provide fermions with correct propagators we introduce righthanded $\psi_{L}^{I}$-partners $\psi_{R}^{I}$ that decouple in four dimensions and are inert under every gauge transformations. We include $\psi_{R}$ and $\bar{\psi}_{R}$ into the set of fields $\Phi$. It is not necessary to introduce sources $K$ for them.

Specifically, we start from the regularized classical action

$$
\begin{aligned}
S_{c \mathrm{r}} & =-\frac{1}{4} \sum_{i} \zeta_{i} \int F_{\mu \nu}^{a_{i}} F^{a_{i} \mu \nu}+\int \bar{\psi}_{L}^{I} \iota \not D \psi_{L}^{I}+S_{\mathrm{LR}} \\
& =S_{c}+S_{\mathrm{LR}},
\end{aligned}
$$

which is the sum of the unregularized classical action (2.1) plus a correction

$S_{\mathrm{LR}}=\varsigma_{I J} \int \bar{\psi}_{R}^{I} \iota \not \partial \psi_{L}^{J}+\varsigma_{J I}^{*} \int \bar{\psi}_{L}^{I} \imath \not \partial \psi_{R}^{J}+\int \bar{\psi}_{R}^{I} \iota \not \partial \psi_{R}^{I}$, where $\varsigma_{I J}$ are constants that form an invertible matrix $\varsigma$. The only nontrivial off-diagonal entries of $\varsigma$ (and of all the matrices $M_{I J}$ we going to meet in this paper) are those that mix equivalent irreducible representations $R_{L}^{I}$. The reason why the matrix $\zeta$ is kept free is that later on it will help us reabsorb the renormalization constants of $\psi_{L}^{I}$, since $S_{\mathrm{LR}}$ is nonrenormalized (see below).

Using the polar decomposition, we can write $\varsigma=$ $U_{R}^{\dagger} D U_{L}$, where $U_{L}$ and $U_{R}$ are unitary matrices and $D$ is a positive-definite diagonal matrix. In the basis where $\zeta$ is replaced by its diagonal form $D \equiv \operatorname{diag}\left(\varsigma_{I}\right)$ the propagators of the Dirac fermions $\psi^{I}=\psi_{L}^{I}+\psi_{R}^{I}$ are

${ }_{l} \delta^{I J} \frac{\not \bar{p}+\varsigma_{I} \hat{p}}{\bar{p}^{2}+\varsigma_{I}^{2} \hat{p}^{2}}$

and coincide with the usual propagators for $\varsigma_{I}=1$.

Next, observe that $\left(S_{K}, S_{K}\right)=0$ in arbitrary $D$. The regularized gauge-fixed action is (up to an extension that will be discussed later)

$S_{r 0}(\Phi, K)=S_{c}+S_{\mathrm{LR}}+\left(S_{K}, \Psi\right)+S_{K}=S_{\mathrm{gf}}+S_{\mathrm{LR}}$,

and satisfies

$$
\begin{array}{r}
\left(S_{r 0}, S_{r 0}\right)=2 \lg \int C^{a}\left(\left(\partial_{\hat{\mu}} \bar{\psi}_{R}^{I}\right) \gamma^{\hat{\mu}} T^{a} \varsigma_{I J} \psi_{L}^{J}\right. \\
\left.+\bar{\psi}_{L}^{I} \varsigma_{J I}^{*} T^{a} \hat{\not} \psi_{R}^{J}\right)=\mathcal{O}(\varepsilon),
\end{array}
$$

where " $\mathcal{O}(\varepsilon)$ " is used to denote any expression that vanishes in four dimensions. We have used $P_{R} \not \partial P_{R}=P_{R} \hat{\not} P_{R}$ and a similar relation with $R \rightarrow L$. Observe that $S_{r 0}$ is invariant under the global symmetry transformations of the group $G$.

Given a (dimensionally) regularized classical action $S(\Phi, K)$, the regularized generating functionals $Z$ and $W$ are defined by the formulas

$$
\begin{aligned}
Z(J, K) & =\int[\mathrm{d} \Phi] \exp \left(\imath S(\Phi, K)+\imath \int \Phi^{\alpha} J_{\alpha}\right) \\
& =\exp \imath W(J, K)
\end{aligned}
$$

and the generating functional $\Gamma(\Phi, K)=W(J, K)-$ $\int \Phi^{\alpha} J_{\alpha}$ of one-particle irreducible diagrams is the Legendre transform of $W(J, K)$ with respect to $J$, where the sources $K$ act as spectators. Often it is necessary to pay attention to the action used to define averages. We denote the averages $\langle\cdots\rangle$ defined by the action $S$ as $\langle\cdots\rangle_{S}$ (at $J_{\alpha} \neq 0$ ). The anomaly functional is

$\mathcal{A}=(\Gamma, \Gamma)=\langle(S, S)\rangle_{S}$

and collects the set of one-particle irreducible correlation functions containing one insertion of $(S, S)$. The last equality of (2.11) can be proved by making the change of field variables $\Phi^{\alpha} \rightarrow \Phi^{\alpha}+\theta\left(S, \Phi^{\alpha}\right)$ inside the functional integral (2.10), where $\theta$ is a constant anticommuting parameter. 
The proof is recalled in Appendix B, together with comments on the meaning of the formula.

No one-particle irreducible diagrams can be constructed with external legs $\bar{\psi}_{R}$ or $\psi_{R}$, because $\bar{\psi}_{R}$ and $\psi_{R}$ do not appear in any vertices. Thus, the total $\Gamma$ functional satisfies

$\Gamma(\Phi, K)=\left.\Gamma(\Phi, K)\right|_{\bar{\psi}_{R}=\psi_{R}=0}+S_{\mathrm{LR}}$.

We have anticipated that the action (2.8) is not the final dimensionally regularized action we are going to use. Before moving to the appropriate extension $S_{r}$, we must describe the counterterms generated by $S_{r 0}$, list a number of properties that may be used to restrict the $S_{r 0}$ extensions and point out some subtleties concerning the dimensional regularization.

First, observe that the counterterms are $B, K_{B}$ and $K_{\bar{C}}$ independent. Indeed, the source $K_{B}$ appears nowhere in $S_{r 0}$, while $K_{\bar{C}}$ appears only in $-\int B K_{\bar{C}}$. Moreover, the gaugefixing conditions are linear in the fields, and the $B$-dependent terms of $S_{r 0}$ are at most quadratic in $\Phi$. Therefore no nontrivial one-particle irreducible diagrams can have external $B$ legs.

Second, the action $S_{r 0}$ does not depend on the antighosts $\bar{C}^{a_{i}}$ and the sources $K^{\mu a_{i}}$ separately, but only through the combinations $K^{\mu a_{i}}+\partial_{\mu} \bar{C}^{a_{i}}$. The $\Gamma$ functional must share the same property. Indeed, an antighost external leg actually carries the structure $\partial_{\mu} \bar{C}^{a_{i}}$, since all vertices containing antighosts do so. Given a diagram with $K^{\mu a_{i}}$ or $\partial_{\mu} \bar{C}^{a_{i}}$ on external legs, we can construct almost identical diagrams by just replacing one or more legs $K^{\mu a_{i}}$ with $\partial_{\mu} \bar{C}^{a_{i}}$, or vice versa.

Third, power counting and ghost-number conservation ensure that the counterterms are linear in the sources $K$. Using square brackets to denote dimensions in units of mass, we have $\left[K^{\mu a}\right]=\left[K_{C}^{a}\right]=2$, and $\left[K_{\psi}\right]=3 / 2$. These sources have negative ghost numbers. Therefore, the dimension of a term that is more than linear in $K$ and has vanishing ghost number necessarily exceeds 4 .

\subsection{Structure of the dependence on the overall gauge coupling}

It is useful to single out how the functionals depend on the overall gauge coupling $g$. The tree-level functionals we work with have the $g$ structure

$X_{\text {tree }}(\Phi, K, g)=\frac{1}{g^{2}} X_{\text {tree }}^{\prime}(g \Phi, g K)$.

If the action satisfies this condition at the tree level, then the renormalized action and the $\Gamma$ functional have the $g$ structure

$X(\Phi, K, g)=\sum_{L \geqslant 0} g^{2(L-1)} X_{L}^{\prime}(g \Phi, g K)$, where $X_{L}$ collects the $L$-loop contributions. Basically, there is an additional factor $g^{2}$ for every loop. Indeed, when the action is of the form (2.12), every vertex is multiplied by a power $g^{N-2}$, where $N$ is the number of its $\Phi$ plus $K$ legs. Then, a one-particle irreducible diagram with $L$ loops, $I$ internal legs, $E$ external legs and $v_{i}$ vertices with $i$ legs is multiplied by

$\prod_{i \geqslant 2} g^{v_{i}(i-2)}=g^{2 I+E-2 V}=g^{E-2} g^{2 L}=g^{E} g^{2(L-1)}$,

having used $L-I+V=1$ and $\sum_{i \geqslant 2} i v_{i}=2 I+E$. We see that for $L \geq 1$ we have one power of $g$ for each external leg and a residual factor $g^{2(L-1)}$, in agreement with (2.13).

The $g$ structures (2.12) and (2.13) are preserved by the antiparentheses: if the functionals $X(\Phi, K, g)$ and $Y(\Phi, K, g)$ satisfy (2.12), or (2.13), then the functional $(X, Y)$ satisfies (2.12), or (2.13), respectively.

\subsection{Properties of the dimensional regularization of chiral theories}

Now we recall a few properties of the dimensional regularization of chiral theories, which are important for the rest of our analysis. It is well known that divergences are just poles in $\varepsilon$. Instead, the terms that disappear when $D \rightarrow 4$, called "evanescences", may be of two types: formal or analytic. Analytically evanescent terms, briefly denoted by "aev", are those that factorize at least one $\varepsilon$, such as $\varepsilon F_{\mu \nu} F^{\mu \nu}, \varepsilon \bar{\psi}_{L} l \not D \psi_{L}$, etc. Formally evanescent terms, briefly denoted by "fev", are those that formally disappear when $D \rightarrow 4$, but do not factorize powers of $\varepsilon$. They are built with the tensor $\delta_{\hat{\mu} \hat{v}}$ and the evanescent components $\hat{x}, \hat{p}, \hat{\partial}$, $\hat{\gamma}, \hat{A}$ of coordinates, momenta, derivatives, gamma matrices and gauge fields. Examples are $\bar{\psi}_{L} l \hat{\partial} \psi_{R},\left(\partial_{\hat{\mu}} A_{v}^{a}\right)\left(\partial^{\hat{\mu}} A^{v a}\right)$, etc.

The distinction between formally evanescent and analytically evanescent expressions is to some extent ambiguous. Consider for example a basis $\bar{\psi}_{1} \gamma^{\rho_{1} \cdots \rho_{k}} \psi_{2}$ of fermion bilinears, where $\psi_{1}, \psi_{2}$ can be $\psi_{L}$ or $K_{\psi}$, and $\gamma^{\rho_{1} \cdots \rho_{k}}$ is the completely antisymmetric product of $\gamma^{\rho_{1}}, \ldots, \gamma^{\rho_{k}}$. In dimensional regularization these bilinears are nonvanishing for every $k$, and they are evanescent for $k>4$. We have several ways to rearrange the products of two or more fermion bilinears by using Fierz identities, and such rearrangements can convert formally evanescent objects into analytically evanescent ones. For example, given some spinors $\psi_{n}, n=1,2,3,4$, we can expand the matrix $\psi_{2} \bar{\psi}_{3}$ in the basis made of $\gamma^{\rho_{1} \cdots \rho_{k}}, k=0, \ldots, \infty$. We have

$\psi_{2} \bar{\psi}_{3}=-\frac{1}{f(D)} \sum_{k=0}^{\infty} \frac{(-1)^{k(k-1) / 2}}{k !} \gamma^{\rho_{1} \cdots \rho_{k}}\left(\bar{\psi}_{3} \gamma_{\rho_{1} \cdots \rho_{k}} \psi_{2}\right)$ 
where $f(D)=\operatorname{tr}[\mathbb{1}]$. Using this identity we find, for example,

$$
\begin{aligned}
& \left(\bar{\psi}_{1} \gamma^{\hat{\mu}} \psi_{2}\right)\left(\bar{\psi}_{3} \gamma_{\hat{\mu}} \psi_{4}\right)=\frac{\varepsilon}{f(D)}\left(\bar{\psi}_{1} \psi_{4}\right)\left(\bar{\psi}_{3} \psi_{2}\right) \\
& -\frac{2}{f(D)}\left(\bar{\psi}_{1} \gamma^{\hat{\rho}} \psi_{4}\right)\left(\bar{\psi}_{3} \gamma_{\hat{\rho}} \psi_{2}\right) \\
& -\frac{\varepsilon}{f(D)}\left(\bar{\psi}_{1} \gamma^{\rho} \psi_{4}\right)\left(\bar{\psi}_{3} \gamma_{\rho} \psi_{2}\right)+\cdots
\end{aligned}
$$

Basically, this equation has the form "fev $=\mathrm{fev}+\mathrm{aev"}$. The existence of such relations poses some problems, which we now describe.

Feynman diagrams may generate "divergent evanescences", briefly denoted by "divev". They are made of products between poles and formal evanescences, such as $\left(\partial_{\hat{\mu}} A_{v}^{a}\right)$ $\left(\partial^{\hat{\mu}} A^{v a}\right) / \varepsilon$. The theorem of locality of counterterms demands that we renormalize divergent evanescences away, together with ordinary divergences (see below). However, this makes sense only if we can define divergent evanescences unambiguously, which could be problematic due to the observations made above. For example, if we multiply both sides of formula (2.14) by $1 / \varepsilon$ we get a relation of the type "divev $=$ finite + divev".

Ultimately, the problem does not arise in the theories we are considering here, for the following reasons. Both the classical action and the counterterms are local functionals, equal to integrals of local functions of dimension 4 . In the paper we also show that the first nonvanishing contributions to the anomaly functional (2.11) are local, equal to integrals of local functions of dimension 5. A fermion bilinear $\bar{\psi}_{1} \gamma^{\rho_{1} \cdots \rho_{k}} \psi_{2}$ has dimension 3, so power counting implies that the classical action, as well as counterterms and local contributions to anomalies, cannot contain products of two or more fermion bilinears. Therefore, they are not affected by the ambiguities discussed above. Those ambiguities can only occur in the convergent sector of the theory, where they are harmless, since both analytic and formal evanescences must eventually disappear.

Thanks to the properties just mentioned, it is meaningful to require that the action $S_{r 0}$, as well as its extensions constructed in the rest of this paper, do not contain analytically evanescent terms. More precisely, the coefficients of every Lagrangian terms should be equal to their four-dimensional limits. This request is important to avoid unwanted simplifications between $\varepsilon$ factors and $\varepsilon$ poles, when divergent parts are extracted from bilinear expressions such as $(\Gamma, \Gamma)$. It can be considered part of the definition of the minimal subtraction scheme. For the same reason, we must be sure that the antiparentheses do not generate extra factors of $\varepsilon$, or poles in $\varepsilon$, which is proved below.

Finite nonevanescent contributions will be called "nev". We need a convention to define these quantities precisely, otherwise they can mix with evanescent terms. For example, we need to state whether $\bar{C} \partial^{2} C$, or $\bar{C} \bar{\partial}^{2} C$, or a combina- tion such as $(1+\alpha \varepsilon) \bar{C} \bar{\partial}^{2} C+\beta \bar{C} \hat{\partial}^{2} C$, where $\alpha$ and $\beta$ are constants, is taken to be nonevanescent. The convention we choose is that nonevanescent terms are maximally symmetric with respect to the $D$-dimensional Lorentz group. For the arguments of this paper we just need to focus on local functionals contributing to counterterms and anomalies. In the case of counterterms the nonevanescent terms are those appearing in the action $S_{r 0}$, which are $\mathrm{SO}(D)$-invariant when chiral fermions are switched off. In the case of anomalies the nonevanescent terms are $\mathrm{SO}(D)$-invariant unless they contain the tensor $\varepsilon^{\mu \nu \rho \sigma}$ or chiral fermions.

\subsection{Evanescent extension of the classical action}

It is convenient to extend the action $S_{r 0}$ by adding all formally evanescent terms that have the features of divergent evanescences, multiplied by independent parameters $\eta$. In this way it is possible to subtract divergent evanescences by means of $\eta$ redefinitions. Denoting the correction collecting such terms with $S_{\mathrm{ev}}$, the extended action reads

$$
\begin{aligned}
S_{r}(\Phi, K)= & S_{r 0}(\Phi, K)+S_{\mathrm{ev}}(\Phi, K)=S_{c}+S_{\mathrm{LR}}+S_{\mathrm{ev}} \\
& +\left(S_{K}, \Psi\right)+S_{K}=S_{\mathrm{gf}}+S_{\mathrm{LR}}+S_{\mathrm{ev}} .
\end{aligned}
$$

Then the generating functionals (2.10), the functional $\Gamma$ and the anomaly functional $\mathcal{A}$ of (2.11) are turned into those defined by $S_{r}$.

Each term of $S_{\mathrm{ev}}$ is the integral of a monomial of dimension $\leqslant 4$, globally invariant under $G$. It not necessarily gauge invariant, since gauge invariance is violated away from four dimensions. Moreover, $S_{\mathrm{ev}}$ is $B, K_{B}, K_{\bar{C}}, \bar{\psi}_{R}$ and $\psi_{R}$ independent, linear in $K$ and depends on $\bar{C}^{a_{i}}$ and the sources $K^{\mu a_{i}}$ only through the combinations $K^{\mu a_{i}}+\partial^{\mu} \bar{C}^{a_{i}}$. It is also independent of $K_{C}, K_{\psi}, \bar{K}_{\psi}, \psi_{L}$ and $\bar{\psi}_{L}$, because no formally evanescent terms can be built with these objects. By power counting and ghost-number conservation the terms proportional to $K^{\mu a_{i}}+\partial^{\mu} \bar{C}^{a_{i}}$ are independent of matter fields. In the end, $S_{\mathrm{ev}}$ has the form

$S_{\mathrm{ev}}(\Phi, K)=S_{c \mathrm{ev}}(A)-\int \sum_{i} R_{\mu \mathrm{ev}}^{a_{i}}(A, C)\left(K^{\mu a_{i}}+\partial^{\mu} \bar{C}^{a_{i}}\right)$.

We can further restrict $S_{\mathrm{ev}}$. Indeed, $S_{r 0}$ satisfies (2.12). Therefore, the divergent evanescences have the form (2.13) with $L \geqslant 1$, and they can be renormalized with an $S_{\mathrm{ev}}$ of the form (2.12). Precisely, we can define the parameters $\eta$ so that $S_{\mathrm{ev}}$ is linear in $\eta$ and its $g$ dependence has the form

$$
\begin{gathered}
S_{\mathrm{ev}}(\Phi, K, g, \eta)=\frac{1}{g^{2}} S_{\mathrm{ev}}^{\prime}(g \Phi, g K, \eta) \equiv \frac{1}{g^{2}} S_{c \mathrm{ev}}^{\prime}(g A, \eta) \\
-\frac{1}{g^{2}} \int \sum_{i} R_{\mu \mathrm{ev}}^{a_{i}^{\prime}}(g A, g C, \eta)\left(g K^{\mu a_{i}}+g \partial^{\mu} \bar{C}^{a_{i}}\right),
\end{gathered}
$$

so $S_{r}$ also satisfies (2.12). 
Basically, the terms of $S_{\mathrm{ev}}$ are similar to those appearing in $S_{r 0}$, but contain some evanescent components of momenta and/or gauge fields, and they are broken into gauge noninvariant pieces. We have

$R_{\mu \mathrm{ev}}^{a_{i}}=\eta_{1 i} \partial_{\hat{\mu}} C^{a_{i}}+\eta_{2 i} g f^{a_{i} b_{i} c_{i}} A_{\hat{\mu}}^{b_{i}} C^{c_{i}}$,

while examples of contributions to $S_{c \text { ev }}$ are

$$
\begin{aligned}
S_{c \text { ev }}= & \sum_{i} \int\left(\eta_{3 i}\left(\partial_{\mu} A_{\hat{\nu}}^{a_{i}}\right)\left(\partial^{\mu} A^{\hat{\nu} a_{i}}\right)+\eta_{4 i}\left(\partial_{\hat{\mu}} A_{\nu}^{a_{i}}\right)\left(\partial^{\hat{\mu}} A^{v a_{i}}\right)\right. \\
& \left.+\eta_{5 i}\left(\partial_{\hat{\mu}} A_{\hat{\nu}}^{a_{i}}\right)\left(\partial^{\hat{\mu}} A^{\hat{\nu} a_{i}}\right)\right) \\
& +\sum_{i} \int\left(\eta_{6 i}\left(\partial_{\hat{\mu}} A^{\hat{\mu} a_{i}}\right)\left(\partial_{\nu} A^{v a_{i}}\right)\right. \\
& \left.+\eta_{7 i}\left(\partial_{\hat{\mu}} A^{\hat{\mu} a_{i}}\right)\left(\partial_{\hat{\nu}} A^{\hat{\nu} a_{i}}\right)+\eta_{8 i} A_{\hat{\mu}}^{a_{i}} A^{\hat{\mu} a_{i}}\right) \\
& +\sum_{i} \int\left(\eta_{9 i} g f^{a_{i} b_{i} c_{i}} A_{\mu}^{a_{i}} A_{\hat{\nu}}^{b_{i}} \partial^{\mu} A^{\hat{\nu} c_{i}}+\cdots\right) \cdot(2.19)
\end{aligned}
$$

The terms multiplied by $\eta_{3 i}, \ldots \eta_{8 i}$ are quadratic and modify the propagators of the gauge fields $A_{\mu}^{a_{i}}$ and the Lagrange multipliers $B^{a_{i}}$. We do not need to report here the modified propagators, which are rather involved. We have checked, with the help of a computer program, that they satisfy the requirements we need. In particular, if $k$ denotes their momentum, (i) they are regular when any evanescent components $\hat{k}$ of $k$ are set to zero; (ii) when the propagators are differentiated with respect to any components $\vec{k}, \hat{k}$, or to parameters of positive dimensions (such as $\eta_{8 i}$ ), their behaviors for large $k^{2}$ improve by at least one power; (iii) they have a regular infrared behavior, which corresponds to the decoupling of the evanescent components $A_{\hat{\mu}}^{a_{i}}$. Finally, their denominators are $\mathrm{SO}(1,3) \times \mathrm{SO}(-\varepsilon)$ scalars, like the denominators of the fermion propagators (2.7).

The extended action (2.15) satisfies

$\left(S_{r}, S_{r}\right)=\left(S_{r 0}, S_{r 0}\right)+\mathcal{O}(\eta) \mathcal{O}(\varepsilon)=\mathcal{O}(\varepsilon)+\mathcal{O}(\eta) \mathcal{O}(\varepsilon)$,

where $\left(S_{r 0}, S_{r 0}\right)$ is given by (2.9).

\subsection{Structure of correlation functions}

Now we analyze the evaluation of correlation functions. We use the same notation for a function and its Fourier transform, since no confusion is expected to arise.

In momentum space, the terms of the classical action can be written in the form

$$
\begin{gathered}
\int\left(\prod_{i=1}^{n+r} \frac{\mathrm{d}^{D} k_{i}}{(2 \pi)^{D}}\right) \Phi^{\alpha_{1}}\left(k_{1}\right) \cdots \Phi^{\alpha_{n}}\left(k_{n}\right) K_{\beta_{1}}\left(k_{n+1}\right) \cdots \\
K_{\beta_{r}}\left(k_{n+r}\right) T_{\mu_{1} \cdots \mu_{p} \alpha_{1} \cdots \alpha_{n}}^{\beta_{1} \cdots \beta_{r}} G^{\mu 1 \cdots \mu_{p}}\left(k_{1}, \ldots, k_{n+r}\right),
\end{gathered}
$$

where $k_{1}, \ldots, k_{n+r}$ are the external momenta.The constants $T_{\mu_{1} \cdots \mu_{p} \alpha_{1} \cdots \alpha_{n}}^{\beta_{1} \cdots \beta_{r}}$ collect all tensors $\eta_{\mu \nu}, \varepsilon_{\mu \nu \rho \sigma}, \delta_{\hat{\mu} \hat{v}}, \gamma$ matri- ces, structure constants $f^{a b c}$ and matrices $T^{a}$. In particular, every projector onto hat components of momenta, fields, and sources is moved inside $T_{\mu_{1} \cdots \mu_{p} \alpha_{1} \cdots \alpha_{n}}^{\beta_{1} \cdots \beta_{r}}$. Momentum conservation ensures that

$$
\begin{aligned}
& G^{\mu_{1} \cdots \mu_{p}}\left(k_{1}, \ldots, k_{n+r}\right) \\
& \quad=(2 \pi)^{D} \delta^{(D)}(P) \tilde{G}^{\mu_{1} \cdots \mu_{p}}\left(k_{1}, \ldots, k_{n+r}\right), \quad P=\sum_{i=1}^{n+r} k_{i},
\end{aligned}
$$

where the tensors $\tilde{G}^{\mu_{1} \cdots \mu_{p}}$ are polynomials that depend on $n+r-1$ external momenta.

Propagators can be decomposed as sums of terms of the form

$T_{\mu_{1} \cdots \mu_{p} \alpha_{1} \alpha_{2}}^{\prime} \frac{N_{\text {prop }}^{\mu_{1} \cdots \mu_{p}}(k)}{D_{\text {prop }}(k)}$,

where $T_{\mu_{1} \cdots \mu_{p} \alpha_{1} \alpha_{2}}^{\prime}$ is a constant tensor, $N_{\text {prop }}^{\mu_{1} \cdots \mu_{p}}(k)$ is a polynomial $\mathrm{SO}(1, D-1)$ tensor, and $D_{\text {prop }}(k)$ is a polynomial $\mathrm{SO}(1,3) \times \mathrm{SO}(-\varepsilon)$ scalar. The reason why $D_{\text {prop }}(k)$ is not fully $\mathrm{SO}(1, D-1)$ invariant is that the regularized propagators do not have $\mathrm{SO}(1, D-1)$-scalar denominators, due to the parameters $\varsigma_{I}$ of (2.7) and the parameters $\eta$ provided by the extension $S_{r 0} \rightarrow S_{r}$ discussed above.

The Feynman diagrams of $\Gamma$ and $\mathcal{A}$ have structures inherited from the structures (2.20) and (2.22) of the vertices and propagators. They may be written as sums of contributions of the form (2.20), with tensors $G^{\mu_{1} \cdots \mu_{p}}$ that satisfy (2.21), but now $\tilde{G}^{\mu_{1} \cdots \mu_{p}}$ are integrals over internal momenta $p$ of rational functions,

$$
\frac{N^{\mu_{1} \cdots \mu_{p}}(p, k)}{D(p, k)}
$$

where the polynomial $N^{\mu_{1} \cdots \mu_{p}}(p, k)$ appearing in the numerator is an $\mathrm{SO}(1, D-1)$ tensor, and the polynomial $D(p, k)$ appearing in the denominator is an $\mathrm{SO}(1,3) \times \mathrm{SO}(-\varepsilon)$ scalar. At $\varsigma_{I J}=\delta_{I J}, \eta=0$ the integrals $\tilde{G}^{\mu_{1} \cdots \mu_{p}}$ are full $\mathrm{SO}(1, D-1)$ tensors. Note that $\tilde{G}^{\mu_{1} \cdots \mu_{p}}$ have a regular limit when the evanescent components $\hat{k}$ of the external momenta $k$ tend to zero.

For example, we can write

$$
\begin{aligned}
& \int \frac{\mathrm{d}^{D} p}{(2 \pi)^{D}} \frac{\left(\hat{p}^{2}\right)^{2}}{\left(\bar{p}^{2}+\varsigma_{I}^{2} \hat{p}^{2}-m^{2}\right)^{2}\left((p-k)^{2}-m^{2}\right)} \\
& =\delta_{\hat{\mu} \hat{\nu}} \delta_{\hat{\rho} \hat{\sigma}} \tilde{G}^{\mu \nu \rho \sigma}(k, m),
\end{aligned}
$$

where

$$
\begin{aligned}
& \tilde{G}^{\mu \nu \rho \sigma}(k, m) \\
& =\int \frac{\mathrm{d}^{D} p}{(2 \pi)^{D}} \frac{p^{\mu} p^{\nu} p^{\rho} p^{\sigma}}{\left(\bar{p}^{2}+\varsigma_{I}^{2} \hat{p}^{2}-m^{2}\right)^{2}\left((p-k)^{2}-m^{2}\right)} .
\end{aligned}
$$


Then we include $\delta_{\hat{\mu} \hat{v}} \delta_{\hat{\rho} \hat{\sigma}}$ inside the constants $T_{\mu_{1} \cdots \mu_{p} \alpha_{1} \cdots \alpha_{n}}^{\beta_{1} \cdots \beta_{r}}$. The remaining completely symmetric tensor $\tilde{G}^{\mu \nu \rho \sigma}(k, m)$ is an integral with the properties listed above.

It may be useful to write (2.20) in the more compact form

$\int L_{\mu_{1} \cdots \mu_{p}}(\Phi, K) G^{\mu_{1} \cdots \mu_{p}}\left(k_{1}, \ldots, k_{n+r}\right)$,

and then organize the expressions $L_{\mu_{1} \cdots \mu_{p}}(\Phi, K)$ by using the basis of fermion bilinears $\bar{\psi}_{1} \gamma^{\rho_{1} \cdots \rho_{k}} \psi_{2}$, and explicitly evaluate traces of spinor indices and contractions of Lorentz indices. At the end, all Lorentz indices appear in gauge fields, fermion bilinears, the tensor $\varepsilon_{\mu \nu \rho \sigma}$ (if present), and $G^{\mu_{1} \cdots \mu_{p}}$, and they are contracted among one another, possibly after projections onto bar or hat components.

It is also convenient to expand

$$
\begin{aligned}
G^{\mu_{1} \cdots \mu_{p}}(k) & =\sum_{i} \Pi_{i}^{\mu_{1} \cdots \mu_{p}}(k) G_{i}(k) \\
& =(2 \pi)^{D} \delta^{(D)}(P) \sum_{i} \Pi_{i}^{\mu_{1} \cdots \mu_{p}}(k) \tilde{G}_{i}(k),
\end{aligned}
$$

where $G_{i}(k)$ and $\tilde{G}_{i}(k)$ are $\mathrm{SO}(1,3) \times \mathrm{SO}(-\varepsilon)$ scalars, and $\Pi_{i}^{\mu_{1} \cdots \mu_{p}}(k)$ are polynomials constructed with $\eta_{\mu \nu}, \varepsilon_{\mu \nu \rho \sigma}$, $\delta_{\hat{\mu} \hat{v}}$ and the $n+r-1$ independent momenta $k$. Then we can write the contribution (2.25) to $\Gamma$ or $\mathcal{A}$ as

$\int L_{i} G^{i}$

where

$L_{i}=L_{\mu_{1} \cdots \mu_{p}}(\Phi, K) \Pi_{i}^{\mu_{1} \cdots \mu_{p}}(k)$

are also $\mathrm{SO}(1,3) \times \mathrm{SO}(-\varepsilon)$ scalars. After these operations, the Lorentz indices appear in gauge fields, fermion bilinears, momenta $k$ and the tensor $\varepsilon_{\mu \nu \rho \sigma}$. They are contracted among themselves, possibly after projections onto bar or hat components. At this point, traces and index contractions must be evaluated explicitly, because they may produce factors $\varepsilon$, which are important for the expansions and limits that we are going to define.

The analytic expansion around $\varepsilon=0$ of (2.25) or (2.27) is defined by expanding the scalars $G^{i}(k)$ in powers of $\varepsilon$ without affecting the evanescent components of external momenta. The analytic limit is the order zero of the analytic expansion, once the poles in $\varepsilon$ have been subtracted away. The formal limit $\varepsilon \rightarrow 0$ is the limit where the evanescent components of gauge fields, external momenta and fermion bilinears are dropped. The limit $\varepsilon \rightarrow 0$ is the analytic limit followed by the formal limit.

For the reasons explained above, the analytic and formal limits may be ambiguous in the convergent sector of the theory, but they are unambiguous in the divergent sector. More importantly, the limit $\varepsilon \rightarrow 0$ is always unambiguous. Since the tensors $G^{\mu_{1} \cdots \mu_{p}}$ are regular when any evanescent compo- nents $\hat{k}$ of the external momenta $k$ are set to zero, the formal limits of (2.25) and (2.27) are well defined.

When we use the expressions " $\mathcal{O}(\varepsilon)$ " or "ev" we mean any quantity that vanishes in the limit $\varepsilon \rightarrow 0$. Clearly, ev $=$ $\mathrm{aev}+\mathrm{fev}$.

\subsection{Locality of counterterms}

Now we comment on the locality of counterterms. The forms of the regularized propagators ensure that a sufficient number of derivatives with respect to physical $\bar{k}$ and/or evanescent $\hat{k}$ components of external momenta $k$ kills the overall divergences of Feynman diagrams. If we subtract the divergent evanescences, together with the ordinary divergences, up to some order $n$, then both ordinary divergences and divergent evanescences of order $n+1$ are polynomial in $\bar{k}$ and $\hat{k}$. The $S_{r 0}$-extension $S_{r}=S_{r 0}+S_{\mathrm{ev}}$ of (2.15) allows us to subtract all of them in a way that is efficient for the proof of the Adler-Bardeen theorem.

To complete the analysis it is useful to describe what happens if for some reason we do not subtract some divergent evanescences. We use the abbreviations "loc" and "nl" to denote local and nonlocal contributions, respectively. At one loop we miss counterterms of the form

$\hbar \frac{\operatorname{loc} \mathrm{fev}}{\varepsilon}$.

Consequently, at two loops we also miss counterterms for subdivergences. Using the vertex (2.28) inside one-loop diagrams we get contributions of the form

$$
\begin{aligned}
& \hbar^{2}\left(\frac{\text { loc nev }}{\varepsilon}+\frac{\text { loc fev }}{\varepsilon}+\mathrm{nl}\right) \\
& +\hbar^{2}\left(\frac{\text { loc fev }}{\varepsilon^{2}}+\frac{\mathrm{nl} \mathrm{fev}}{\varepsilon}+\mathrm{nl} \mathrm{fev}\right) .
\end{aligned}
$$

The first three terms are generated when the formal evanescence enters the diagram, is converted into a factor $\varepsilon$ and simplifies a pole in $\varepsilon$. Symbolically, we express this occurrence (which is the basic mechanism that originates potential anomalies) as

fev $\cap$ one-loop $\rightarrow \hbar$ (loc nev + loc fev $+\mathcal{O}(\varepsilon)$ nl).

The last three terms of (2.29) describe what happens when the formal evanescence remains outside the diagram.

The first term of (2.29) must be subtracted, so the missing counterterms at two loops are

$\hbar^{2} \frac{\text { loc fev }}{\varepsilon^{2}}, \quad \hbar^{2} \frac{\text { loc fev }}{\varepsilon}, \quad \hbar^{2} \frac{\mathrm{nl} \mathrm{fev}}{\varepsilon}$.

Even if the last term of this list is nonlocal, we still have no problem, since the residues of the poles in $\varepsilon$ are formally evanescent. However, when we use the first and third terms of (2.31) inside one-loop diagrams, the formal evanescence 
can simplify another pole, by the mechanism (2.30), and give

$\hbar^{3} \frac{\mathrm{nl} \mathrm{nev}}{\varepsilon}+\hbar^{3} \frac{\mathrm{nl} \mathrm{fev}}{\varepsilon^{2}}+\hbar^{3} \frac{\mathrm{nl} \mathrm{fev}}{\varepsilon}+\hbar^{3} \mathrm{nl}$

plus local poles. We see that nonlocal, nonevanescent divergences appear at three loops. These are only partially compensated by analogous contributions originating by the subtraction of the first term of (2.29). Those due to the first term of (2.31), in particular, do not seem to disappear.

On the other hand, it is safe to subtract the divergent evanescences order by order, together with nonevanescent divergences. In this paper we adopt this prescription.

\subsection{Properties of the antiparentheses}

Now we study how divergences and evanescences propagate through the antiparentheses. Indeed, in the proofs of renormalizability to all orders and the Adler-Bardeen theorem, it is necessary to extract divergent parts of antiparentheses such as $\mathcal{A}=(\Gamma, \Gamma)$ or $(\Gamma, \mathcal{A})$. This operation is not as simple as it sounds, because we must be sure that the antiparentheses themselves do not generate poles or factors of $\varepsilon$, in order to be able to say that, for example, the divergent part of $\left(S_{r}, \Gamma^{(1)}\right)$ is equal to $\left(S_{r}, \Gamma_{\text {div }}^{(1)}\right)$, where $\Gamma^{(1)}$ it the one-loop contribution to $\Gamma$ and $\Gamma_{\text {div }}^{(1)}$ is the divergent part of $\Gamma^{(1)}$. Specifically, we prove that

(i) the antiparentheses ( $\left.X_{\text {conv }}, Y_{\text {conv }}\right)$ of convergent functionals $X_{\text {conv }}$ and $Y_{\text {conv }}$ are convergent;

(ii) the antiparentheses $\left(X_{\text {conv }}, Y_{\mathrm{ev}}\right)$ of convergent functionals $X_{\text {conv }}$ and evanescent functionals $Y_{\mathrm{ev}}$ are evanescent;

(iii) the antiparentheses $(X, Y)$ do not generate either poles in $\varepsilon$ or factors of $\varepsilon$ if $X, Y$ and $(X, Y)$ do not involve products of two or more fermion bilinears.

For the use we have in mind it is convenient to rephrase property (iii) more explicitly as

(iii') the antiparentheses $\left(X_{\mathrm{A}}, Y_{\mathrm{B}}\right)$ of functionals $X_{\mathrm{A}}$ and $Y_{\mathrm{B}}$ with the properties specified by their subscripts $\mathrm{A}$ and $\mathrm{B}$, satisfy the identities

$$
\begin{aligned}
& \left(X_{\mathrm{fev}}, Y_{\mathrm{nev} / \mathrm{fev}}\right)=\mathrm{fev}, \quad\left(X_{\mathrm{divev}}, Y_{\text {nev } / \mathrm{fev} / \mathrm{divev}}\right)=\mathrm{divev}, \\
& \left(X_{\mathrm{ev}}, Y_{\mathrm{fev}}\right)=\mathrm{ev},\left.\quad\left(X_{\mathrm{nev}}, Y_{\mathrm{div}}\right)\right|_{\mathrm{div}}=\left(X_{\mathrm{nev}}, Y_{\mathrm{div}}\right), \\
& \left.\left(X_{\mathrm{nev}}, Y_{\text {nev }}\right)\right|_{\text {nev }}=\left(X_{\text {nev }}, Y_{\text {nev }}\right) \\
& \left.\left(X_{\mathrm{nev}}, Y_{\text {nev div }}\right)\right|_{\text {nev div }}=\left(X_{\text {nev }}, Y_{\text {nev div }}\right),
\end{aligned}
$$

as long as $X_{\mathrm{A}}, Y_{\mathrm{B}}$ and $\left(X_{\mathrm{A}}, Y_{\mathrm{B}}\right)$ donot involve products of two or more fermion bilinears.

To prove these properties it is convenient to write the antiparentheses in momentum space. We have

$$
\int \mathrm{d}^{D} x \frac{\delta_{r} X}{\delta \Phi^{\alpha}(x)} \frac{\delta_{l} Y}{\delta K_{\alpha}(x)}=\int \frac{\mathrm{d}^{D} p}{(2 \pi)^{D}} \frac{\delta_{r} X}{\delta \Phi^{\alpha}(p)} \frac{\delta_{l} Y}{\delta K_{\alpha}(-p)}
$$

and a similar relation obtained by exchanging $\Phi$ and $K$. Let us write (2.27) for $X, Y$ and $(X, Y)$ as

$X=\int L_{i X} G_{X}^{i}, \quad Y=\int L_{j Y} G_{Y}^{j}, \quad(X, Y)=\int L_{i j(X, Y)} G_{X Y}^{i j}$.

Using (2.26) we find that the $p$ integral of (2.33) can be readily done and gives

$G_{X Y}^{i j}=(2 \pi)^{D} \delta^{(D)}(P) \tilde{G}_{X}^{i} \tilde{G}_{Y}^{j}$,

where $P$ is the total momentum of $\tilde{G}_{X}^{i}$ plus the ones of $\tilde{G}_{Y}^{j}$. We see that the scalar "cores" $G^{i}$ of correlation functions just multiply each other in momentum space, which cannot generate new poles in $\varepsilon$ or factors of $\varepsilon$.

It remains to study the relation between $L_{i j(X, Y)}$ and $L_{i X}$, $L_{j Y}$. The antiparentheses can produce index contractions by means of the paired functional derivatives $\delta / \delta A_{\mu}-\delta / \delta K^{\mu}$ and $\delta / \delta \psi-\delta / \delta K_{\psi}$. Clearly, no such operations can generate poles in $\varepsilon$. This observation is sufficient to prove statements (i) and (ii).

As far as statement (iii) is concerned, we must assume that the functionals $X, Y$ and $(X, Y)$ do not involve products of two or more fermion bilinears. Therefore, they are free of ambiguities of type (2.14). The contraction of Lorentz indices brought about by $\delta / \delta A_{\mu}$ and $\delta / \delta K^{\mu}$ gives a tensor $\eta^{\mu \nu}$ with mixed indices (namely one index from $X$ and one index from $Y$ ). The contraction of spinorial indices brought about by $\delta / \delta \psi$ and $\delta / \delta K_{\psi}$ gives structures such as

$\bar{\psi}_{1} \gamma^{\rho_{1} \cdots \rho_{k}} \gamma^{\sigma_{1} \cdots \sigma_{l}} \psi_{2}$,

where the $\rho$ indices come from $X$ and the $\sigma$ indices come from $Y$. Anticommuting the $\gamma$ 's we can rearrange the indices so that $\rho_{1}<\rho_{2}<\cdots<\rho_{k}$ and $\sigma_{1}<\sigma_{2}<\cdots<\sigma_{l}$. Reordering the indices we may get minus signs from further anticommutations or from squares of $\gamma$ matrices with identical indices. In the end, we get a formula like

$$
\begin{aligned}
& \bar{\psi}_{1} \gamma^{\rho_{1} \cdots \rho_{k}} \gamma^{\sigma_{1} \cdots \sigma_{l}} \psi_{2} \\
& \quad=\sum \pm \bar{\psi}_{1} \gamma^{\rho_{1} \cdots \breve{\rho}_{m} \cdots \rho_{k} \sigma_{1} \cdots \breve{\sigma}_{n} \cdots \sigma_{l}} \psi_{2} \prod \eta^{\rho_{m} \sigma_{n}},
\end{aligned}
$$

where the breves denote missing indices that go into the tensors $\eta^{\mu \nu}$. Again, we get only tensors $\eta^{\mu \nu}$ with mixed indices. We recall that all Lorentz indices, possibly after projection onto bar or hat components, are contracted with gauge fields, fermion bilinears, momenta and possibly $\varepsilon_{\mu \nu \rho \sigma}$, and that, by assumption, no products of two or more fermion bilinears are involved. Then it is obvious that the contractions originating by the antiparentheses cannot produce $\varepsilon$ factors. Using these properties it is easy to check that identities (2.32) hold, so statement (iii) is also proved. 
Statement (iii) also says that the antiparentheses cannot convert formal $\varepsilon$ evanescences into analytic ones. It applies, for example, to local functionals $X$ and $Y$ that are equal to the integrals of functions of dimensions $n_{X}, n_{Y} \leqslant 5$, such that $n_{X}+n_{Y} \leqslant 8$, because then $X, Y$ and $(X, Y)$ cannot contain products of two or more fermion bilinears. In the paper we will apply statement (iii) to the divergent contributions to $\Gamma$ and the first nonvanishing contributions to the anomaly functional $\mathcal{A}$ of (3.10).

\section{DHD regularization}

The dimensional regularization alone does not provide the subtraction scheme where the cancellation of gauge anomalies is manifest to all orders. To find the right scheme, we modify the regularization technique by adding higherderivative terms that preserve gauge invariance in $D=4$. We take the non-gauge-fixed regularized classical action

$$
\begin{aligned}
S_{c \Lambda}= & S_{c}+S_{\mathrm{LR}}-\frac{1}{4} \int F_{\mu \nu}^{a}\left(\frac{D^{2}}{\Lambda^{2}}\right)^{8} F^{a \mu \nu} \\
& +\sum_{I} \int \bar{\psi}_{L}^{I} l \not D\left(\frac{D^{2}}{\Lambda^{2}}\right)^{3} \psi_{L}^{I}+S_{\Lambda \mathrm{LR}},
\end{aligned}
$$

where

$$
\begin{aligned}
S_{\Lambda \mathrm{LR}}= & \sum_{I} \int \bar{\psi}^{I} \iota \not \partial\left(\frac{\partial^{2}}{\Lambda^{2}}\right)^{3} \psi^{I} \\
& -\sum_{I} \int \bar{\psi}_{L}^{I} \iota \not \partial\left(\frac{\partial^{2}}{\Lambda^{2}}\right)^{3} \psi_{L}^{I} .
\end{aligned}
$$

The higher-derivative structures of (3.1) and (3.2) are chosen to simplify the arguments of our derivations.

We gauge fix $S_{c \Lambda}$ by using modified gauge-fixing functions of the form

$\mathcal{G}_{\Lambda}^{a_{i}}=Q(\square) \partial^{\mu} A_{\mu}^{a_{i}}, \quad Q(\square)=1+\frac{\lambda^{\prime}}{\Lambda^{16}} \square^{8}$,

and a modified gauge fermion

$$
\begin{aligned}
\Psi_{\Lambda}(\Phi) & =\sum_{i} \int \bar{C}^{a_{i}}\left(\mathcal{G}_{\Lambda}^{a_{i}}+\frac{1}{2} P_{i}(\square) B^{a_{i}}\right), \\
P_{i}(\square) & =\xi_{i}+\frac{\xi^{\prime}}{\Lambda^{16}} \square^{8},
\end{aligned}
$$

where $\lambda^{\prime}$ and $\xi^{\prime}$ are other (dimensionless) gauge-fixing parameters.

Finally, we add

$$
\begin{aligned}
S_{\Lambda \mathrm{ev}}= & S_{c \mathrm{ev}}(A)-\int \sum_{i} R_{\mu \mathrm{ev}}^{a_{i}}(A, C) \\
& \times\left(K^{\mu a_{i}}+Q(\square) \partial^{\mu} \bar{C}^{a_{i}}\right),
\end{aligned}
$$

which differs from $S_{\mathrm{ev}}$ only because the combinations $K^{\mu a_{i}}+\partial^{\mu} \bar{C}^{a_{i}}$ are replaced by $K^{\mu a_{i}}+Q(\square) \partial^{\mu} \bar{C}^{a_{i}}$.

The regularized gauge-fixed action reads

$S_{\Lambda}(\Phi, K)=S_{c \Lambda}+S_{\Lambda \mathrm{ev}}+\left(S_{K}, \Psi_{\Lambda}\right)+S_{K}$,

where $S_{K}$ is the same as before, and satisfies

$$
\begin{aligned}
\left(S_{\Lambda}, S_{\Lambda}\right)= & 2 g \int C^{a}\left(\left(\partial_{\hat{\mu}} h_{I J}\left(\partial^{2}\right) \bar{\psi}_{R}^{I}\right) l \gamma^{\hat{\mu}} T^{a} \psi_{L}^{J}\right. \\
& \left.+\bar{\psi}_{L}^{I} T^{a} \imath \hat{\not} h_{J I}^{*}\left(\partial^{2}\right) \psi_{R}^{J}\right)+\mathcal{O}(\eta) \mathcal{O}(\varepsilon),
\end{aligned}
$$

where $h_{I J}\left(\partial^{2}\right)=\left(\varsigma_{I J} \Lambda^{6}+\delta_{I J}\left(\partial^{2}\right)^{3}\right) / \Lambda^{6}$. The reason why it is useful to separate the terms proportional to the parameters $\eta$ will become clear later.

It is straightforward to derive the propagators and check that the ones of gauge fields, $\left\langle A_{\mu}(k) A_{\nu}(-k)\right\rangle_{0}$, and the ones of ghosts, $\langle C(k) \bar{C}(-k)\rangle_{0}$, fall off as $1 /\left(k^{2}\right)^{9}$ for large momenta $k$, while the propagators $\langle A(k) B(-k)\rangle_{0}$ fall off as $k /\left(k^{2}\right)^{9}$, and $\langle B(k) B(-k)\rangle_{0}$ as $1 /\left(k^{2}\right)^{8}$. For example, in the "Feynman gauge" $\xi_{i}=\lambda^{\prime}=\xi^{\prime}=1$ at $\eta=0$ we have

$$
\begin{aligned}
\left\langle A_{\mu}(k) A_{\nu}(-k)\right\rangle_{0} & =-\frac{l \eta_{\mu \nu}}{k^{2} Q\left(-k^{2}\right)}, \\
\langle C(k) \bar{C}(-k)\rangle_{0} & =\frac{l}{k^{2} Q\left(-k^{2}\right)} .
\end{aligned}
$$

The fermion propagators, on the other hand, fall off as $p /\left(p^{2}\right)^{4}$.

For a while we need to work at finite $\Lambda$, where the action $S_{\Lambda}$ is super-renormalizable. To make its superrenormalizability manifest, it is convenient to parametrize it so that the $\Lambda$ denominators cancel out. Let us first ignore the terms $S_{\Lambda \text { ev }}$. We define tilde fields and tilde parameters as $\tilde{A}_{\mu}^{a}=\frac{A_{\mu}^{a}}{\Lambda^{8}}, \quad \tilde{\psi}^{I}=\frac{\psi^{I}}{\Lambda^{3}}, \quad \tilde{g}=\Lambda^{8} g, \quad \tilde{\zeta}_{i}=\Lambda^{16} \zeta_{i}$,

and $\tilde{r}_{i}=r_{i}$. The covariant derivatives remain $\Lambda$ independent. To cancel the $\Lambda$ denominators of the gauge-fixing sector we define $\widetilde{\bar{C}}^{a}=\bar{C}^{a} / \Lambda^{8}, \tilde{B}^{a}=B^{a} / \Lambda^{8}$ and $\tilde{C}^{a}=C^{a} / \Lambda^{8}$. Finally, we define the tilde sources

$$
\begin{aligned}
& \left(\tilde{K}^{\mu a}, \tilde{K}_{C}^{a}, \tilde{K}_{\bar{C}}^{a}, \tilde{K}_{B}^{a}, \tilde{K}_{\psi}^{I}, \tilde{\bar{K}}_{\psi}^{I}\right) \\
& =\left(\Lambda^{8} K^{\mu a}, \Lambda^{8} K_{C}^{a}, \Lambda^{8} K_{\bar{C}}^{a}, \Lambda^{8} K_{B}^{a}, \Lambda^{3} K_{\psi}^{I}, \Lambda^{3} \bar{K}_{\psi}^{I}\right),
\end{aligned}
$$

so the tilde map is a canonical transformation combined with a redefinition of parameters.

As far as $S_{\Lambda \mathrm{ev}}$ is concerned, using (2.17) and the linearity in $\eta$ we can write it as

$$
\begin{aligned}
& \tilde{S}_{\Lambda \mathrm{ev}}=\frac{1}{\tilde{g}^{2}} S_{c \mathrm{ev}}^{\prime}\left(\tilde{g} \tilde{A}, \Lambda^{16} \eta\right) \\
& -\frac{1}{\tilde{g}^{2}} \sum_{i} \int R_{\mu \mathrm{ev}}^{a_{i}^{\prime}}(\tilde{g} \tilde{A}, \tilde{g} \tilde{C}, \eta)\left(\tilde{g} \tilde{K}^{\mu a_{i}}+\tilde{g} \tilde{Q}(\square) \partial^{\mu} \widetilde{\bar{C}}^{a_{i}}\right),
\end{aligned}
$$


where $\tilde{Q}(\square)=\Lambda^{16}+\lambda^{\prime} \square^{8}$.

In the tilde parametrization the full action reads

$$
\begin{aligned}
& \tilde{S}_{\Lambda}(\tilde{\Phi}, \tilde{K}) \equiv S_{\Lambda}(\Phi(\tilde{\Phi}), K(\tilde{K})) \\
& =-\frac{1}{4} \sum_{i} \int \tilde{F}_{\mu \nu}^{a_{i}}\left(\tilde{\zeta}_{i}+\left(\tilde{D}^{2}\right)^{8}\right) \tilde{F}^{a_{i} \mu \nu} \\
& \quad+\int \widetilde{\bar{\psi}}_{L}^{I} \iota \tilde{D}\left(\Lambda^{6}+\left(\tilde{D}^{2}\right)^{3}\right) \tilde{\psi}_{L}^{I} \\
& \quad+\int \widetilde{\bar{\psi}}_{R}^{I} l \not \partial\left(\Lambda^{6}+\left(\partial^{2}\right)^{3}\right) \tilde{\psi}_{R}^{I} \\
& \quad+\int \widetilde{\bar{\psi}}_{R}^{I} l \not \partial\left(\varsigma_{I J} \Lambda^{6}+\delta_{I J}\left(\partial^{2}\right)^{3}\right) \tilde{\psi}_{L}^{J} \\
& \quad+\int \widetilde{\bar{\psi}}_{L}^{I} \iota \not \partial\left(\varsigma_{J I}^{*} \Lambda^{6}+\delta_{I J}\left(\partial^{2}\right)^{3}\right) \tilde{\psi}_{R}^{J} \\
& \quad+\sum_{i} \int \tilde{B}^{a_{i}} \tilde{Q}(\square) \partial^{\mu} \tilde{A}_{\mu}^{a_{i}}+\frac{1}{2} \sum_{i} \int \tilde{B}^{a_{i}} \tilde{P}_{i}(\square) \tilde{B}^{a_{i}} \\
& \quad-\sum_{i} \int \widetilde{\bar{C}}^{a_{i}} \tilde{Q}(\square) \partial^{\mu} \tilde{D}_{\mu} \tilde{C}^{a_{i}} \\
& \quad-\int R^{\alpha}(\tilde{\Phi}, \tilde{g}) \tilde{K}_{\alpha}+\tilde{S}_{\Lambda \mathrm{ev}},
\end{aligned}
$$

where $\tilde{P}_{i}(\square)=\tilde{\xi}_{i}+\xi^{\prime} \square^{8}, \tilde{\xi}_{i}=\xi_{i} \Lambda^{16}$.

The DHD-regularized generating functional $Z_{\Lambda}$ reads

$$
\begin{aligned}
Z_{\Lambda}(J, K) & =\int[\mathrm{d} \Phi] \exp \left(\imath S_{\Lambda}(\Phi, K)+\imath \int \Phi^{\alpha} J_{\alpha}\right) \\
& =\exp \imath W_{\Lambda}(J, K),
\end{aligned}
$$

and the generating functional $\Gamma_{\Lambda}(\Phi, K)=W_{\Lambda}(J, K)-$ $\int \Phi^{\alpha} J_{\alpha}$ of one-particle irreducible diagrams is the Legendre transform of $W_{\Lambda}(J, K)$ with respect to $J$. Since no oneparticle irreducible diagrams with external legs $\psi_{R}, \bar{\psi}_{R}$ can be constructed, the action $S_{\Lambda}$ and the $\Gamma$ functional $\Gamma_{\Lambda}$ depend on $\psi_{R}, \bar{\psi}_{R}$ in exactly the same way. The DHD-regularized anomaly functional is

$\mathcal{A}_{\Lambda}=\left(\Gamma_{\Lambda}, \Gamma_{\Lambda}\right)=\left\langle\left(S_{\Lambda}, S_{\Lambda}\right)\right\rangle_{S_{\Lambda}}$.

When we switch to the tilde parametrization we write $\tilde{Z}_{\Lambda}$, $\tilde{W}_{\Lambda}, \tilde{\Gamma}_{\Lambda}$ and $\tilde{\mathcal{A}}_{\Lambda}$. See appendix B for the proof of the last equality of (3.10).

The tilde action $\tilde{S}_{\Lambda}$ is polynomial in $\Lambda$, has properly normalized propagators and contains only parameters of nonnegative dimensions in units of mass. However, the tilde fields have negative dimensions, which in principle may jeopardize the (super)renormalizability we want to prove. Precisely, we have

$[\tilde{A}]=[\tilde{\bar{C}}]=[\tilde{C}]=-7, \quad[\tilde{B}]=-6, \quad[\tilde{\psi}]=-\frac{3}{2}, \quad[\tilde{g}]=8$,

while $\left[\tilde{K}^{a \mu}\right]=\left[\tilde{K}_{C}^{a}\right]=\left[\tilde{K}_{\bar{C}}^{a}\right]=10,\left[\tilde{K}_{B}\right]=9$ and $\left[\tilde{K}_{\psi}\right]=$ $9 / 2$. The problem is solved as follows. Since $S_{\Lambda}$ has the form (2.12), the $\tilde{g}$ structure of $\tilde{S}_{\Lambda}$ is the tilde version of (2.12). The tilde version of (2.13) ensures that the counterterms have the $\tilde{g}$ structure

$\sum_{L \geqslant 1} \tilde{g}^{2(L-1)} F_{L}(\tilde{g} \tilde{\Phi}, \tilde{g} \tilde{K})$,

where the $L$-loop local functionals $F_{L}$ depend polynomially on the other dimensionful parameters of the theory. Then we see that the theory is indeed super-renormalizable, because the dimensions of all products $\tilde{g} \tilde{\Phi}$ and $\tilde{g} \tilde{K}$ are strictly positive.

\subsection{The DHD limit}

The basic idea behind the DHD regularization is to "first send $\varepsilon$ to zero, then $\Lambda$ to infinity". However, we must formulate the rules of such limits more precisely, since certain caveats demand attention. We distinguish the higherderivative theory from the final theory. The higher-derivative theory is the one defined by the classical action $S_{\Lambda}$ (or $\tilde{S}_{\Lambda}$, if we use the tilde parametrization), where the scale $\Lambda$ is kept fixed and treated like any other parameter, instead of a cutoff. It is super-renormalizable and regularized by the dimensional technique. Its divergences, which are poles in $\varepsilon$, are subtracted in the next section using the minimal subtraction scheme. The final theory is obtained by taking the limit $\Lambda \rightarrow \infty$ on the renormalized higher-derivative theory, after subtracting the $\Lambda$ divergences that emerge in that limit.

Having already expanded in $\varepsilon$, we may wonder what types of divergences appear in the final theory. We have products $\Lambda^{k} \ln ^{k^{\prime}} \Lambda$ of powers and logarithms of $\Lambda$, but we also have terms that are evanescent in $\varepsilon$ and divergent in $\Lambda$. To understand what to do with these, we distinguish two types of them, according to whether the $\varepsilon$ evanescence is analytic or formal.

(i) First, consider analytic evanescences in $\varepsilon$ multiplied by products $\Lambda^{k} \ln ^{k^{\prime}} \Lambda$, such as $\varepsilon \Lambda^{2} \ln \Lambda$. Since we first send $\varepsilon$ to zero, these quantities are not true divergences and must be neglected. In any case, they cannot be subtracted away, because the theorem of locality of counterterms does not apply to them. Consider for example the integral

$$
\begin{aligned}
& \int \frac{\mathrm{d}^{D} p}{(2 \pi)^{D}} \frac{\Lambda^{4}}{\left(p^{2}+m^{2}\right)\left(\Lambda^{4}+\left(p^{2}\right)^{2}\right)} \\
& =\frac{\Lambda^{4-\varepsilon} m^{2}\left[\cos \left(\frac{\pi \varepsilon}{4}\right)+\frac{\Lambda^{2}}{m^{2}} \sin \left(\frac{\pi \varepsilon}{4}\right)-\frac{\Lambda^{\varepsilon}}{m^{\varepsilon}}\right]}{2^{D} \pi^{(D-2) / 2} \Gamma\left(\frac{D}{2}\right)\left(\Lambda^{4}+m^{4}\right) \sin \left(\frac{\pi \varepsilon}{2}\right)},
\end{aligned}
$$

where for the purposes of our present discussion the mass $m$ can also play the role of an external momentum. Expanding the right-hand side in powers of $\varepsilon$ we find that the $\mathcal{O}\left(\varepsilon^{0}\right)$ terms, which are equal to 
$\frac{1}{32 \pi^{2}}\left(\pi \Lambda^{2}-2 m^{2} \ln \frac{\Lambda^{2}}{m^{2}}\right)+\mathcal{O}\left(\frac{m}{\Lambda}\right)$,

have a $\Lambda$-divergent part that is polynomial in $m$, as expected, while the $\mathcal{O}\left(\varepsilon^{1}\right)$ terms have a $\Lambda$-divergent part that contains expressions such as

$$
\Lambda^{2} \ln \frac{\Lambda^{2}}{m^{2}}, \quad m^{2} \ln ^{2} \frac{\Lambda^{2}}{m^{2}}
$$

which are not polynomial in $m$.

(ii) Next, consider formal evanescences times $\Lambda^{k} \ln ^{k^{\prime}} \Lambda$, such as $(\ln \Lambda) \partial_{\mu} A_{\hat{v}} \partial^{\mu} A^{\hat{v}}$. These can (actually, must, for the reasons explained in Sect. 2.5) be subtracted away (as long as their coefficients are calculated at $\varepsilon=0$ ), because the form of regularized propagators ensures that counterterms are polynomial in both physical and evanescent components of external momenta and fields.

(iii) Formally evanescent expressions multiplied by products $\Lambda^{k} \ln ^{k^{\prime}} \Lambda$ and factors of $\varepsilon$ are just like case (i) and should not be subtracted away.

(iv) For completeness, we point out a fourth type of $\varepsilon$ evanescent $\Lambda$ divergences, that is to say, nonlocal contributions of type (ii), which can appear as artifacts of inconvenient manipulations. Precisely, because of the ambiguities encoded in (2.14) some quantities of type (i) can be converted into nonlocal divergences of type (ii). These conversions should just be avoided. To this purpose, it is sufficient to note that the structure (2.20) of diagrams and the expansion of the integrals $G^{\mu_{1} \cdots \mu_{p}}$ only generate $\varepsilon$-evanescent $\Lambda$ divergences of types (i), (ii) and (iii). In the event that "aev $\rightarrow$ fev conversions" of type (2.14) are accidentally applied, nonlocal divergences of type (ii) can just be ignored, because they cannot mix with the local terms belonging to the power-counting renormalizable sector and they are resummable into contributions of type (i).

To summarize, the $\Lambda$ divergences are equal to $\Lambda^{k} \ln ^{k^{\prime}} \Lambda$ times local monomials of the fields, the sources and their derivatives. From the point of view of the dimensional regularization, those monomials may be nonevanescent or formally evanescent, and their coefficients must be evaluated in the analytic limit $\varepsilon \rightarrow 0$.

We can thus define the procedure with which we renormalize the final theory and define the physical quantities. We call it the DHD limit. We still organize the contributions to $\Gamma$ and $\mathcal{A}$ in the form (2.20). Referring to (2.25) and (2.27), the DHD limit is made of the analytic limit $\varepsilon \rightarrow 0$, followed by the limit $\Lambda \rightarrow \infty$, followed by the formal limit $\varepsilon \rightarrow 0$. We also have the $D H D$ expansion, that is to say, the analytic expansion around $\varepsilon=0$ followed by the expansion around $\Lambda=\infty$.
The three steps that define the DHD limit are unambiguous in the divergent sector, which does not contain products of more than one fermion bilinears. Instead, the first and third steps are ambiguous in the convergent sector. What is important is that the DHD limit is also unambiguous in the convergent sector.

It is useful to recapitulate the DHD limit in symbolic form. We first expand around $\varepsilon=0$ at $\Lambda$ fixed, and find poles, finite terms and evanescent terms:

$$
\frac{1}{\varepsilon}, \quad \frac{\hat{\delta}}{\varepsilon}, \quad \varepsilon^{0}, \quad \hat{\delta} \varepsilon^{0}, \quad \varepsilon, \quad \hat{\delta} \varepsilon .
$$

The symbols appearing in this list have the following meanings: $1 / \varepsilon$ denotes any kinds of divergences in $\varepsilon, \hat{\delta}$ is any formally evanescent quantity, $\varepsilon^{0}$ is any quantity that is convergent and nonevanescent in the analytic limit $\varepsilon \rightarrow 0$, and $\varepsilon$ denotes any analytic evanescence. After the expansion, we subtract the poles and remain with

$\varepsilon^{0}, \quad \hat{\delta} \varepsilon^{0}, \quad \varepsilon, \quad \hat{\delta} \varepsilon$.

The terms proportional to $\varepsilon$ vanish in the DHD limit. The terms $\hat{\delta} \varepsilon^{0}$ also vanish in that limit, but for some time we treat them together with the $\varepsilon^{0}$ terms. Next, we study the $\Lambda$ dependence. Expanding the coefficients of every surviving terms (3.12) around $\Lambda=\infty$, we find

$$
\begin{aligned}
& \varepsilon^{0} \Lambda, \quad \hat{\delta} \varepsilon^{0} \Lambda, \quad \varepsilon^{0} \Lambda^{0}, \quad \hat{\delta} \varepsilon^{0} \Lambda^{0}, \quad \frac{\varepsilon^{0}}{\Lambda}, \quad \frac{\hat{\delta} \varepsilon^{0}}{\Lambda}, \\
& \varepsilon \Lambda, \quad \hat{\delta} \varepsilon \Lambda, \quad \varepsilon \Lambda^{0}, \quad \hat{\delta} \varepsilon \Lambda^{0}, \quad \frac{\varepsilon}{\Lambda}, \quad \frac{\hat{\delta} \varepsilon}{\Lambda},
\end{aligned}
$$

where $\Lambda$ denotes any kind of $\Lambda$-divergent expression (such as $\Lambda^{k} \ln ^{k^{\prime}} \Lambda$, with $k, k^{\prime} \geqslant 0$ and $k+k^{\prime}>0$ ), while $\Lambda^{0}$ is any $\Lambda$ convergent, non- $\Lambda$-evanescent expression, and $1 / \Lambda$ is any $\Lambda$ -evanescent expression. Then we subtract the $\Lambda$ divergences of the DHD limit, namely the terms of types $\varepsilon^{0} \Lambda$ and $\hat{\delta} \varepsilon^{0} \Lambda$. After that we remain with

$$
\begin{aligned}
& \varepsilon^{0} \Lambda^{0}, \quad \hat{\delta} \varepsilon^{0} \Lambda^{0}, \quad \frac{\varepsilon^{0}}{\Lambda}, \quad \frac{\hat{\delta} \varepsilon^{0}}{\Lambda}, \quad \varepsilon \Lambda, \\
& \hat{\delta} \varepsilon \Lambda, \quad \varepsilon \Lambda^{0}, \quad \hat{\delta} \varepsilon \Lambda^{0}, \quad \frac{\varepsilon}{\Lambda}, \quad \frac{\hat{\delta} \varepsilon}{\Lambda} .
\end{aligned}
$$

At this point we are ready to take the DHD limit, which drops all contributions of this list but the $\varepsilon^{0} \Lambda^{0}$ terms.

\section{Renormalization of the higher-derivative theory}

In this section and the next two we study the higher-derivative regularized theory $\tilde{S}_{\Lambda}$, keeping $\Lambda$ fixed and (mostly) using the tilde parametrization. We first work out the renormalization of the theory, then study its one-loop anomalies and finally prove the anomaly cancellation to all orders. 
The counterterms (3.11) are local and largely constrained. We know that (i) they are independent of $\tilde{B}, \tilde{K}_{\bar{C}}, \tilde{K}_{B}$, $\tilde{\psi}_{R}$ and $\widetilde{\bar{\psi}}_{R}$ and (ii) do not depend on antighosts $\widetilde{\widetilde{C}}^{a_{i}}$ and sources $\tilde{K}^{\mu a_{i}}$ separately, but only through the combinations $\tilde{K}^{\mu a_{i}}+\tilde{Q}(\square) \partial^{\mu} \widetilde{\bar{C}}^{a_{i}}$. Indeed, we have arranged $S_{\Lambda \text { ev }}$ to preserve these properties. Actually, we have chosen the higherderivative structure of $S_{\Lambda}$ to simplify the counterterms even more: (iii) they cannot depend on the sources $\tilde{K}$ and matter fields $\tilde{\psi}$, because each product $\tilde{g} \tilde{K}, \tilde{g} \tilde{\psi}$ has dimension greater than 4; (iv) they cannot contain antighosts, because of points (ii) and (iii); (v) they cannot contain ghosts, because all objects with negative ghost numbers are excluded by points (iii) and (iv); (vi) they can only be one-loop, because each loop carries an extra factor $\tilde{g}^{2}$, which has dimension 16 . In the end, there can only be one-loop divergences of the form

$\partial^{2}(\tilde{g} \tilde{A})^{2}, \quad \partial(\tilde{g} \tilde{A})^{3}, \quad(\tilde{g} \tilde{A})^{4}$

(where derivatives can act on any objects to their right), and those obtained from these expressions by suppressing some $\tilde{g} \tilde{A}$ 's or derivatives.

The anomaly functional (3.10), if nonvanishing and nontrivial (in a sense specified below), is the anomaly of the higher-derivative theory. In the tilde parametrization we have

$\tilde{\mathcal{A}}_{\Lambda}=\left(\tilde{\Gamma}_{\Lambda}, \tilde{\Gamma}_{\Lambda}\right)=\left\langle\left(\tilde{S}_{\Lambda}, \tilde{S}_{\Lambda}\right)\right\rangle_{\tilde{S}_{\Lambda}}$.

The one-loop contribution $\tilde{\mathcal{A}}_{\Lambda}^{(1)}$ is

$\tilde{\mathcal{A}}_{\Lambda}^{(1)}=2\left(\tilde{S}_{\Lambda}, \tilde{\Gamma}_{\Lambda}^{(1)}\right)=\left.\left\langle\left(\tilde{S}_{\Lambda}, \tilde{S}_{\Lambda}\right)\right\rangle_{\tilde{S}_{\Lambda}}\right|_{\text {one-loop }}$,

where $\tilde{\Gamma}_{\Lambda}^{(1)}$ is the one-loop contribution to $\tilde{\Gamma}_{\Lambda}$. Using (2.32) and (3.4) we see that $\left(\tilde{S}_{\Lambda}, \tilde{S}_{\Lambda}\right)=$ fev. The right-hand side of (4.3) collects one-loop Feynman diagrams containing insertions of formally evanescent vertices. The formal evanescences can: (a) remain attached to external legs and momenta, or (b) be turned into one or more factors $\varepsilon$. In case (a) they give local divergent evanescences plus nonlocal evanescences. In case (b) the factors $\varepsilon$ can simplify a local divergent part and give local nonevanescent contributions, in addition to (generically nonlocal) evanescences. Therefore, we can write

$\tilde{\mathcal{A}}_{\Lambda}^{(1)}=\tilde{\mathcal{A}}_{\Lambda \text { nev }}^{(1)}+\tilde{\mathcal{A}}_{\Lambda \text { divev }}^{(1)}+\tilde{\mathcal{A}}_{\Lambda \text { ev }}^{(1)}$,

where $\tilde{\mathcal{A}}_{\Lambda \text { nev }}^{(1)}$ is local, convergent, and nonevanescent, $\tilde{\mathcal{A}}_{\Lambda \text { divev }}^{(1)}$ is local and divergent-evanescent and $\tilde{\mathcal{A}}_{\Lambda \text { ev }}^{(1)}$ is evanescent and possibly nonlocal.

Now we take the divergent part of equation (4.3). Decompose $\tilde{\Gamma}_{\Lambda}^{(1)}$ as the sum of its divergent part $\tilde{\Gamma}_{\Lambda \text { div }}^{(1)}$ and its convergent part $\tilde{\Gamma}_{\Lambda \text { conv }}^{(1)}$. Recalling that the antiparentheses of convergent functionals are convergent, we see that $\left(\tilde{S}_{\Lambda}, \tilde{\Gamma}_{\Lambda \text { conv }}^{(1)}\right)$ is convergent. The properties (2.32) apply to $\left(\tilde{S}_{\Lambda}, \tilde{\Gamma}_{\Lambda \text { div }}^{(1)}\right)$, so we have the identity
$\left(\tilde{S}_{\Lambda}, \tilde{\Gamma}_{\Lambda \text { div }}^{(1)}\right)=\frac{1}{2} \tilde{\mathcal{A}}_{\Lambda \text { divev }}^{(1)}$.

Now, (4.1) tells us that $\tilde{\Gamma}_{\Lambda \text { div }}^{(1)}$ is just a functional of $\tilde{g} \tilde{A}$. Therefore, its antiparentheses with $\tilde{S}_{\Lambda}$ are only sensitive to $\tilde{S}_{K}$ and the $K$-dependent contributions to $\tilde{S}_{\Lambda \text { ev }}$, which we denote by $\tilde{S}_{\Lambda K \text { ev }}$. Moreover, we can further decompose $\tilde{\Gamma}_{\Lambda \text { div }}^{(1)}$ as the sum of a nonevanescent divergent part $\tilde{\Gamma}_{\Lambda \text { nevdiv }}^{(1)}$ and a divergent evanescence $\tilde{\Gamma}_{\Lambda \text { divev }}^{(1)}$. So doing we find

$\left(\tilde{S}_{K}+\tilde{S}_{\Lambda K \text { ev }}, \tilde{\Gamma}_{\Lambda \text { nev div }}^{(1)}+\tilde{\Gamma}_{\Lambda \text { divev }}^{(1)}\right)=\frac{1}{2} \tilde{\mathcal{A}}_{\Lambda \text { divev }}^{(1)}$.

At this point, taking the nonevanescent divergent part of this equation, we obtain

$\left(\tilde{S}_{K}, \tilde{\Gamma}_{\Lambda \text { nevdiv }}^{(1)}\right)=0$,

which just states that $\tilde{\Gamma}_{\Lambda \text { nev div }}^{(1)}$ is gauge invariant. Going back to the nontilde parametrization, we have $\tilde{\Gamma}_{\Lambda \text { nev div }}^{(1)}(\tilde{g} \tilde{A})=$ $\Gamma_{\Lambda \text { nevdiv }}^{(1)}(g A)$. By power counting, $\Gamma_{\Lambda \text { nevdiv }}^{(1)}$ can only be a linear combination of the invariants $F_{\mu \nu}^{a_{i}} F^{a_{i}} \mu \nu$, and it can be subtracted by redefining the parameters $\zeta_{i}$. The rest, $\Gamma_{\Lambda \text { divev }}^{(1)}$, can be subtracted by redefining the parameters $\eta$ of $S_{\mathrm{ev}}$. The renormalized action $\hat{S}_{\Lambda}$ is obtained by making the replacements

$\zeta_{i} \rightarrow \zeta_{i}+\frac{f_{i}}{\varepsilon} g^{2}, \quad \eta \rightarrow \eta+\frac{f^{\prime}}{\varepsilon} g^{2}$,

in $S_{\Lambda}$, where $f_{i}, f^{\prime}$ are calculable numerical coefficients. Since $S_{\Lambda}$ is linear in $\zeta$ and $\eta$, we have

$\hat{S}_{\Lambda}=\tilde{S}_{\Lambda}-\tilde{\Gamma}_{\Lambda \operatorname{div}}^{(1)}$.

Moreover, using (4.5) and $\left(\tilde{\Gamma}_{\Lambda \text { div }}^{(1)}, \tilde{\Gamma}_{\Lambda \text { div }}^{(1)}\right)=0$ we find

$\left(\hat{S}_{\Lambda}, \hat{S}_{\Lambda}\right)=\left(\tilde{S}_{\Lambda}, \tilde{S}_{\Lambda}\right)-\tilde{\mathcal{A}}_{\Lambda \text { divev }}^{(1)} \cdot$

The generating functional $\hat{\Gamma}_{\Lambda}$ defined by $\hat{S}_{\Lambda}$ is convergent to all orders, because (3.11) ensures that no divergences can appear beyond one loop. Finally, $\hat{\Gamma}_{\Lambda}$ and the anomaly $\hat{\mathcal{A}}_{\Lambda}=\left(\hat{\Gamma}_{\Lambda}, \hat{\Gamma}_{\Lambda}\right)$ are obtained by making the replacements (4.7) inside $\tilde{\Gamma}_{\Lambda}$ and $\tilde{\mathcal{A}}_{\Lambda}=\left(\tilde{\Gamma}_{\Lambda}, \tilde{\Gamma}_{\Lambda}\right)$, respectively. Clearly, $\hat{\mathcal{A}}_{\Lambda}$ is convergent, because $\hat{\Gamma}_{\Lambda}$ is convergent, and because the antiparentheses of convergent functionals are convergent.

\section{One-loop anomalies}

In this section we study the one-loop anomalies, and relate those of the final theory, which are trivial by assumption, to those of the higher-derivative theory, which turn out to be trivial as a consequence. 
We begin with the one-loop contributions $\hat{\mathcal{A}}_{\Lambda}^{(1)}$ and $\tilde{\mathcal{A}}_{\Lambda}^{(1)}$ to $\hat{\mathcal{A}}_{\Lambda}$ and $\tilde{\mathcal{A}}_{\Lambda}$. First, we observe that

$$
\begin{aligned}
\hat{\mathcal{A}}_{\Lambda} & =\left\langle\left(\hat{S}_{\Lambda}, \hat{S}_{\Lambda}\right)\right\rangle_{\hat{S}_{\Lambda}}=\left\langle\left(\hat{S}_{\Lambda}, \hat{S}_{\Lambda}\right)\right\rangle_{\tilde{S}_{\Lambda}-\tilde{\Gamma}_{\Lambda \mathrm{div}}^{(1)}} \\
& =\left\langle\left(\hat{S}_{\Lambda}, \hat{S}_{\Lambda}\right)\right\rangle_{\tilde{S}_{\Lambda}}+\mathcal{O}\left(\hbar^{2}\right) .
\end{aligned}
$$

Indeed, the correction $\tilde{\Gamma}_{\Lambda \text { div }}^{(1)}$ to the action provides $\mathcal{O}(\hbar)$ vertices. If we use those vertices in one-particle irreducible diagrams together with vertices of $\left(\hat{S}_{\Lambda}, \hat{S}_{\Lambda}\right)$, we must close at least one loop, which gives $\mathcal{O}\left(\hbar^{2}\right)$ contributions. Using (4.9), we have

$$
\begin{aligned}
\hat{\mathcal{A}}_{\Lambda} & =\left\langle\left(\tilde{S}_{\Lambda}, \tilde{S}_{\Lambda}\right)\right\rangle_{\tilde{S}_{\Lambda}}-\tilde{\mathcal{A}}_{\Lambda \text { divev }}^{(1)}+\mathcal{O}\left(\hbar^{2}\right) \\
& =\tilde{\mathcal{A}}_{\Lambda}-\tilde{\mathcal{A}}_{\Lambda \text { divev }}^{(1)}+\mathcal{O}\left(\hbar^{2}\right)
\end{aligned}
$$

thus (4.4) gives

$\hat{\mathcal{A}}_{\Lambda}^{(1)}=\tilde{\mathcal{A}}_{\Lambda \mathrm{nev}}^{(1)}+\tilde{\mathcal{A}}_{\Lambda \mathrm{ev}}^{(1)}$.

As a check, recall that $\hat{\mathcal{A}}_{\Lambda}$ is convergent, so the divergent evanescences $\tilde{\mathcal{A}}_{\Lambda \text { divev }}^{(1)}$ must disappear from $\hat{\mathcal{A}}_{\Lambda}^{(1)}$.

We know that $\tilde{\mathcal{A}}_{\Lambda \text { nev }}^{(1)}$ is the integral of a local function of dimension 5 and ghost number 1 . Recalling that a factor $\tilde{g}$ is attached to every external leg, we have

$\tilde{\mathcal{A}}_{\Lambda \mathrm{nev}}^{(1)}=\int \mathrm{d}^{D} x \tilde{g}^{a} \tilde{\mathcal{A}}^{a}(\tilde{g} \tilde{\Phi}, \tilde{g} \tilde{K})$,

where $\tilde{\mathcal{A}}^{a}$ are local functions of ghost number zero and dimension 4. However, $\tilde{\mathcal{A}}_{\Lambda \text { nev }}^{(1)}$ cannot depend on the sources $\tilde{K}$ and the matter fields $\tilde{\psi}$, because the products $\tilde{g} \tilde{K}$ and $\tilde{g} \tilde{\psi}$ have dimensions greater than 4.

Working out $\left(\tilde{S}_{\Lambda}, \tilde{S}_{\Lambda}\right)$ in detail, it is easy to check that it does not depend on $\tilde{B}^{a_{i}}$ and depends on $\tilde{K}^{\mu a_{i}}$ and $\widetilde{\bar{C}}^{a_{i}}$ only through the combinations $\tilde{K}^{\mu a_{i}}+\tilde{Q}(\square) \partial^{\mu} \widetilde{\widetilde{C}}^{a_{i}}$. Therefore, the same must be true of $\tilde{\mathcal{A}}_{\Lambda}^{(1)}$, which means that $\tilde{\mathcal{A}}_{\Lambda \text { nev }}^{(1)}$ cannot depend on either $\widetilde{\bar{C}}$ or $\tilde{B}$. Then the functions $\tilde{\mathcal{A}}^{a}$ cannot even contain ghosts. Summarizing, we can write

$\tilde{\mathcal{A}}_{\Lambda \mathrm{nev}}^{(1)}=\int \mathrm{d}^{D} x \tilde{g} \tilde{C}^{a} \tilde{\mathcal{A}}^{a}(\tilde{g} \tilde{A})$.

Recall that the antiparentheses satisfy the identity $(X,(X, X))=0$ for any functional $X$. Taking $X=\hat{\Gamma}_{\Lambda}$, we obtain

$\left(\hat{\Gamma}_{\Lambda}, \hat{\mathcal{A}}_{\Lambda}\right)=0$

which are the Wess-Zumino consistency conditions [10], written using the Batalin-Vilkovisky formalism. In particular, at one loop we have

$\left(\tilde{S}_{\Lambda}, \hat{\mathcal{A}}_{\Lambda}^{(1)}\right)=-\left(\hat{\Gamma}_{\Lambda}^{(1)},\left(\tilde{S}_{\Lambda}, \tilde{S}_{\Lambda}\right)\right)$

In Sect. 2 we have proved that the antiparentheses of an evanescent functional with a convergent functional are evanescent. Thus,
$\left(\hat{\Gamma}_{\Lambda}^{(1)},\left(\tilde{S}_{\Lambda}, \tilde{S}_{\Lambda}\right)\right)=\mathrm{ev}=\mathcal{O}(\varepsilon)$.

For the same reason, $\left(\tilde{S}_{\Lambda}, \tilde{\mathcal{A}}_{\Lambda \text { ev }}^{(1)}\right)$ and $\left(\tilde{S}_{\Lambda K \text { ev }}, \tilde{\mathcal{A}}_{\Lambda \text { nev }}^{(1)}\right)$ are evanescent. Using these facts, together with (5.1) and (5.3), (5.5) gives

$$
\begin{aligned}
\mathrm{ev} & =\left(\tilde{S}_{\Lambda}, \tilde{\mathcal{A}}_{\Lambda \mathrm{nev}}^{(1)}+\tilde{\mathcal{A}}_{\Lambda \mathrm{ev}}^{(1)}\right)=\left(\tilde{S}_{\Lambda}, \tilde{\mathcal{A}}_{\Lambda \mathrm{nev}}^{(1)}\right)+\mathrm{ev} \\
& =\left(\tilde{S}_{K}+\tilde{S}_{\Lambda K \mathrm{ev}}, \tilde{\mathcal{A}}_{\Lambda \mathrm{nev}}^{(1)}\right)+\mathrm{ev}=\left(\tilde{S}_{K}, \tilde{\mathcal{A}}_{\Lambda \mathrm{nev}}^{(1)}\right)+\mathrm{ev} .
\end{aligned}
$$

At this point, we take the nonevanescent part of both sides and note that the relations $(2.32)$ apply to $\left(\tilde{S}_{K}, \tilde{\mathcal{A}}_{\Lambda \text { nev }}^{(1)}\right)$, because, thanks to (5.3), no products of more fermion bilinears are involved in these antiparentheses. We find

$\left(\tilde{S}_{K}, \tilde{\mathcal{A}}_{\Lambda \text { nev }}^{(1)}\right)=0$.

Now, $\tilde{\mathcal{A}}_{\Lambda \text { nev }}^{(1)}$ is the (potential) one-loop anomaly of the higher-derivative regularized theory $\tilde{S}_{\Lambda}$, defined keeping $\Lambda$ fixed. The final theory is instead obtained taking the DHD limit. We must relate $\tilde{\mathcal{A}}_{\Lambda \text { nev }}^{(1)}$ to the potential one-loop anomaly $\mathcal{A}_{f \text { nev }}^{(1)}$ of the final theory. Indeed, we are assuming that $\mathcal{A}_{f \text { nev }}^{(1)}$ is trivial (the final theory cannot have gauge anomalies at one loop), but we have no information of this type as regards $\tilde{\mathcal{A}}_{\Lambda \text { nev }}^{(1)}$.

We know how $\tilde{\mathcal{A}}_{\Lambda \text { nev }}^{(1)}$ depends on $\tilde{g}$. The other dimensionful parameters of $\tilde{S}_{\Lambda}$ (such as $\tilde{\zeta}_{i}$ and $\tilde{\xi}_{i}$ ), as well as the powers of $\Lambda$ multiplying various terms (such as $\widetilde{\bar{\psi}}_{L}^{I} \widetilde{D}^{\prime} \tilde{\psi}_{L}^{I}$ ), have dimensions greater than 4 . They cannot contribute to $\tilde{\mathcal{A}}_{\Lambda \text { nev }}^{(1)}$, because the local functions $\tilde{\mathcal{A}}^{a}$ are polynomial in them and have dimension 4 . Thus, $\tilde{\mathcal{A}}_{\Lambda \text { nev }}^{(1)}$ can only depend on $\tilde{g} \tilde{C}, \tilde{g} \tilde{A}, \tilde{r}_{i}, \lambda^{\prime}, \xi^{\prime}, \eta_{1 i}$ and $\eta_{2 i}$. Using (5.3), switching to nontilde variables, and recalling that $\tilde{g} \tilde{A}=g A, \tilde{g} \tilde{C}=g C$, we see that $\mathcal{A}_{\Lambda \text { nev }}^{(1)}$ is $\Lambda$ independent. Now we show that actually $\mathcal{A}_{\Lambda \text { nev }}^{(1)}$ coincides with the one-loop anomaly $\mathcal{A}_{f \text { nev }}^{(1)}$ of the final theory.

To prove this fact, we need to take $\Lambda$ to infinity and study the DHD limit at one loop. A more comprehensive study of the DHD limit will be carried out later. The terms that are divergent in this limit are denoted by "Ddiv", to distinguish them from the divergences considered so far, which strictly speaking were " $\varepsilon$ div". Recall that, according to the definition of DHD limit, the $\Lambda$-divergent parts cannot contain analytic $\varepsilon$ evanescences, but can contain formal $\varepsilon$ evanescences.

Consider $\hat{\mathcal{A}}_{\Lambda}=\left(\hat{\Gamma}_{\Lambda}, \hat{\Gamma}_{\Lambda}\right)$ and take the one-loop DHDdivergent part of this equation. Using (5.1) and recalling that $\mathcal{A}_{\Lambda \text { nev }}^{(1)}$ is $\Lambda$ independent, we get

$$
\begin{aligned}
\left.\frac{1}{2} \mathcal{A}_{\Lambda \text { ev }}^{(1)}\right|_{\text {Ddiv }}= & \left.\left(S_{\Lambda}, \hat{\Gamma}_{\Lambda}^{(1)}\right)\right|_{\text {Ddiv }}=\left.\left(S_{\Lambda}, \hat{\Gamma}_{\Lambda \text { Ddiv }}^{(1)}\right)\right|_{\text {Ddiv }} \\
= & \left.\left(S_{\Lambda}-S_{r}, \hat{\Gamma}_{\Lambda \text { Ddiv }}^{(1)}\right)\right|_{\text {Ddiv }} \\
& +\left.\left(S_{r}, \hat{\Gamma}_{\Lambda \text { Ddiv }}^{(1)}\right)\right|_{\text {Ddiv }}=\left(S_{r}, \hat{\Gamma}_{\Lambda \text { Ddiv }}^{(1)}\right)
\end{aligned}
$$


where $\hat{\Gamma}_{\Lambda \text { Ddiv }}^{(1)}$ is the one-loop DHD-divergent part of $\hat{\Gamma}_{\Lambda}$. In the last step we have dropped the contribution involving $\left(S_{\Lambda}-S_{r}, \hat{\Gamma}_{\Lambda \text { Ddiv }}^{(1)}\right)$, since this quantity vanishes in the limit $\Lambda \rightarrow \infty$. The reason is that, by formulas (2.15) and (3.4), the difference $S_{\Lambda}-S_{r}$ is made of $\mathcal{O}\left(1 / \Lambda^{6}\right)$ terms, and the powerlike $\Lambda$ divergences contained in $\hat{\Gamma}_{\Lambda \text { Ddiv }}^{(1)}$ cannot exceed $\Lambda^{4}$. Actually, this is one of the reasons why we have chosen the particular higher-derivative structure of the theory $S_{\Lambda}$. Moreover, to make the last step of (5.7) we have applied (2.32) to $\left(S_{r}, \hat{\Gamma}_{\Lambda \text { Ddiv }}^{(1)}\right)$. Because of the analysis of Sect. 3 , the $\Lambda$ divergences of $\hat{\Gamma}_{\Lambda \text { Ddiv }}^{(1)}$ may be of two types, with respect to the limit $\varepsilon \rightarrow 0$ : nonevanescent or formally evanescent. Thanks to (2.32), the antiparentheses with $S_{r}$ also give nonevanescent or formally evanescent contributions, wherefrom the last equality of (5.7) follows.

Subtracting the $\Lambda$ divergences $\hat{\Gamma}_{\Lambda \text { Ddiv }}^{(1)}$ from $\hat{S}_{\Lambda}$, we can define the one-loop renormalized action $\hat{S}_{f \text { ren }}$ of the final theory, which reads

$\hat{S}_{f \text { ren }}=\hat{S}_{\Lambda}-\hat{\Gamma}_{\Lambda \text { Ddiv }}^{(1)}+\mathcal{O}\left(\hbar^{2}\right)$.

For the moment we do not need to specify the $\mathcal{O}\left(\hbar^{2}\right)$ terms of this subtraction (but later we will have to be precise about them). The anomaly of the final theory is

$\mathcal{A}_{f}=\left\langle\left(\hat{S}_{f \text { ren }}, \hat{S}_{f \text { ren }}\right)\right\rangle_{\hat{S}_{f \text { ren }}}$,

and its one-loop nonevanescent part is the quantity $\mathcal{A}_{f \text { nev }}^{(1)}$ we want, where the subscript "nev" close to the subscript " $f$ " denotes the contributions that do not vanish in the DHD limit. We have

$$
\begin{aligned}
\mathcal{A}_{f}= & \left\langle\left(\hat{S}_{\Lambda}-\hat{\Gamma}_{\Lambda \text { Ddiv }}^{(1)}, \hat{S}_{\Lambda}-\hat{\Gamma}_{\Lambda \text { Ddiv }}^{(1)}\right)\right\rangle_{\hat{S}_{\Lambda}-\hat{\Gamma}_{\Lambda \text { Ddiv }}^{(1)}+\mathcal{O}\left(\hbar^{2}\right)} \\
= & \hat{\mathcal{A}}_{\Lambda}-2\left(S_{\Lambda}, \hat{\Gamma}_{\Lambda \text { Ddiv }}^{(1)}\right)+\mathcal{O}\left(\hbar^{2}\right) \\
= & \left(S_{\Lambda}, S_{\Lambda}\right)+\mathcal{A}_{\Lambda \text { nev }}^{(1)}+\mathcal{A}_{\Lambda \text { ev }}^{(1)}-2\left(S_{r}, \hat{\Gamma}_{\Lambda \text { Ddiv }}^{(1)}\right) \\
& -2\left(S_{\Lambda}-S_{r}, \hat{\Gamma}_{\Lambda \text { Ddiv }}^{(1)}\right)+\mathcal{O}\left(\hbar^{2}\right) .
\end{aligned}
$$

In these manipulations we have used the formula

$$
\hat{\mathcal{A}}_{\Lambda}=\left\langle\left(\hat{S}_{\Lambda}, \hat{S}_{\Lambda}\right)\right\rangle_{\hat{S}_{\Lambda}}=\left\langle\left(\hat{S}_{\Lambda}, \hat{S}_{\Lambda}\right)\right\rangle_{\hat{S}_{\Lambda}-\hat{\Gamma}_{\Lambda \text { Ddiv }}^{(1)}}+\mathcal{O}\left(\hbar^{2}\right),
$$

which holds because at one loop the vertices of $\hat{\Gamma}_{\Lambda \text { Ddiv }}^{(1)}$, which are already $\mathcal{O}(\hbar)$, cannot contribute to one-particle irreducible diagrams containing one insertion of $\left(\hat{S}_{\Lambda}, \hat{S}_{\Lambda}\right)$.

At one loop, using (5.7), we obtain

$$
\begin{aligned}
\mathcal{A}_{f}^{(1)}= & \mathcal{A}_{\Lambda \mathrm{nev}}^{(1)}+\mathcal{A}_{\Lambda \mathrm{ev}}^{(1)}-\left.\mathcal{A}_{\Lambda \mathrm{ev}}^{(1)}\right|_{\mathrm{Ddiv}} \\
& -2\left(S_{\Lambda}-S_{r}, \hat{\Gamma}_{\Lambda \mathrm{Ddiv}}^{(1)}\right) .
\end{aligned}
$$

We are ready to take the DHD limit. Recall that $\left(S_{\Lambda}-\right.$ $S_{r}, \hat{\Gamma}_{\Lambda \text { Ddiv }}^{(1)}$ ) tends to zero for $\Lambda \rightarrow \infty$, while $\mathcal{A}_{\Lambda \text { nev }}^{(1)}$ does not change. On the other hand, $\mathcal{A}_{\Lambda \text { ev }}^{(1)}$ and its $\Lambda$-divergent part do not separately tend to zero, because they can contain (local) terms that are formally $\varepsilon$ evanescent and $\Lambda$ divergent. However, those terms are precisely $\left.\mathcal{A}_{\Lambda \text { ev }}^{(1)}\right|_{\text {Ddiv }}$, so they disappear in the difference $\mathcal{A}_{\Lambda \text { ev }}^{(1)}-\left.\mathcal{A}_{\Lambda \text { ev }}^{(1)}\right|_{\text {Ddiv }}$. Finally, using (5.3), we get

$\mathcal{A}_{f \text { nev }}^{(1)}=\mathcal{A}_{\Lambda \text { nev }}^{(1)}=\int \mathrm{d}^{D} x g C^{a} \mathcal{A}^{a}(g A)$,

as we wanted.

Let us write the most general structure of the functions $\mathcal{A}^{a}(\mathrm{~g} A)$. We know that they have dimension 4 and are sums of terms of the form $g^{p} \partial^{k} A^{p}$. Power counting gives $k+p \leq 4$, hence we have

$\mathcal{A}^{a} \sim g^{2} \partial^{2} A^{2}+g^{3} \partial A^{3}+g^{4} A^{4}$,

plus the terms obtained from these by suppressing some $g A$ 's or some derivatives. Now it remains to collect all pieces of information found so far and solve (5.6). We call condition (5.6) a little cohomological problem, because it involves a structure (5.3) that contains a finite number of terms, in our case just a few, and its solution can be worked out directly. We recall the solution without proof, because the proof is well known and not necessary for the other derivations of this paper. The solution can be split into the sum of trivial and nontrivial contributions. Trivial contributions are those of the form $\left(S_{K}, \chi\right)$, where $\chi=\chi(g A)$ is a local functional of the gauge fields $A$, equal to the integral of a local function of dimension 4 and ghost number 0 , and having a $g$ structure corresponding to the one-loop sector of (2.13). In the tilde parametrization, we write $\chi$ as $\tilde{\chi}(\tilde{g} \tilde{A})$. The only nontrivial contributions to $\mathcal{A}_{f \text { nev }}^{(1)}$ are proportional to the famous Bardeen formula [18]. In appendix A, the coefficient of the Bardeen term is calculated using our regularization technique. In the end, we have

$$
\begin{aligned}
& \mathcal{A}_{f \mathrm{nev}}^{(1)}=\mathcal{A}_{\Lambda \mathrm{nev}}^{(1)}=-\frac{l g^{3}}{12 \pi^{2}} \int \mathrm{d}^{D} x \varepsilon^{\mu \nu \rho \sigma} \\
& \quad \times \operatorname{Tr}\left[\partial_{\mu} C\left(A_{\nu} \partial_{\rho} A_{\sigma}+\frac{g}{2} A_{\nu} A_{\rho} A_{\sigma}\right)\right]+\left(S_{K}, \chi\right),
\end{aligned}
$$

where $C=C^{a} T^{a}, A_{\mu}=A_{\mu}^{a} T^{a}$, the Bardeen term being the integral on the right-hand side.

One-loop gauge anomalies vanish when the trace appearing in (5.11) vanishes. Typically, the cancellation is possible when the gauge group is a product group and the theory contains various types of fermionic fields in suitable representations, as in the standard model.

Now we go back to the higher-derivative theory (the DHD limit being completed in Sect. 7), precisely to the classical action $\hat{S}_{\Lambda}$ of (4.8). The trivial contributions $\left(S_{K}, \chi\right)$ to the anomalies can be canceled out by redefining the action as

$\hat{S}_{\Lambda}^{\prime}(\Phi, K)=\hat{S}_{\Lambda}(\Phi, K)-\frac{1}{2} \chi(g A)$, 
because then

$$
\begin{aligned}
\hat{\mathcal{A}}_{\Lambda}^{\prime} & =\left\langle\left(\hat{S}_{\Lambda}^{\prime}, \hat{S}_{\Lambda}^{\prime}\right)\right\rangle_{\hat{S}_{\Lambda}^{\prime}}=\left\langle\left(\hat{S}_{\Lambda}, \hat{S}_{\Lambda}\right)\right\rangle_{\hat{S}_{\Lambda}}-\left(\hat{S}_{\Lambda}, \chi\right)+\mathcal{O}\left(\hbar^{2}\right) \\
& =\hat{\mathcal{A}}_{\Lambda}-\left(S_{K}+S_{\Lambda K \mathrm{ev}}, \chi\right)+\mathcal{O}\left(\hbar^{2}\right) .
\end{aligned}
$$

In the last step we used the fact that $\chi$ is $K$ independent. Thus, at one loop we have

$\hat{\mathcal{A}}_{\Lambda}^{\prime(1)}=\mathcal{A}_{\Lambda \text { nev }}^{(1)}+\mathcal{A}_{\Lambda \mathrm{ev}}^{(1)}-\left(S_{K}, \chi\right)+\mathrm{ev}$,

$\hat{\mathcal{A}}_{\Lambda \mathrm{nev}}^{\prime(1)}=\mathcal{A}_{\Lambda \mathrm{nev}}^{(1)}-\left(S_{K}, \chi\right)$,

which means that when the Bardeen term vanishes $\hat{\mathcal{A}}_{\Lambda \text { nev }}^{\prime(1)}=$ $0, \hat{\mathcal{A}}_{\Lambda}^{\prime(1)}=$ ev.

Finally, observe that the new $\Gamma$ functional $\hat{\Gamma}_{\Lambda}^{\prime}$ is still convergent to all orders. The reason is that it is convergent at one loop and the action

$\hat{S}_{\Lambda}^{\prime}=S_{\Lambda}-\Gamma_{\Lambda \text { div }}^{(1)}-\frac{1}{2} \chi$

has the $g$ structure (2.13). Then, using tilde variables, the counterterms must have the form (3.11), which, however, forbids divergent contributions from two loops onwards. The anomaly functional $\hat{\mathcal{A}}_{\Lambda}^{\prime}=\left(\hat{\Gamma}_{\Lambda}^{\prime}, \hat{\Gamma}_{\Lambda}^{\prime}\right)$ is also convergent to all orders and has the $g$ structure (2.13).

The next step is to prove the anomaly cancellation to all orders in the higher-derivative theory. After that, we will have to complete the DHD limit by renormalizing the $\Lambda$ divergences.

\section{Manifest Adler-Bardeen theorem in the higher-derivative theory}

In this section we prove that gauge anomalies manifestly cancel to all orders in the higher-derivative theory $S_{\Lambda}$. We assume that the final theory has no one-loop anomalies, which, according to the previous section, implies that the higherderivative theory shares the same property, namely $\mathcal{A}_{\Lambda \text { nev }}^{(1)}=$ $\left(S_{K}, \chi\right), \hat{\mathcal{A}}_{\Lambda \text { nev }}^{\prime(1)}=0$. Then, the one-loop contribution $\hat{\mathcal{A}}_{\Lambda}^{\prime(1)}$ to the anomaly functional $\hat{\mathcal{A}}_{\Lambda}^{\prime}$ is evanescent, so we can write

$\hat{\mathcal{A}}_{\Lambda}^{\prime}=\mathcal{O}(\varepsilon)+\mathcal{O}\left(\hbar^{2}\right)$.

Here " $\mathcal{O}(\varepsilon)$ " includes the tree-level contribution $\left(S_{\Lambda}, S_{\Lambda}\right)$.

Now we move on to higher orders. We have to study the diagrams with two or more loops, and one insertion of

$$
\begin{aligned}
\mathcal{E} \equiv & \left(\hat{S}_{\Lambda}^{\prime}, \hat{S}_{\Lambda}^{\prime}\right)=\left(\tilde{S}_{\Lambda}, \tilde{S}_{\Lambda}\right)-\tilde{\mathcal{A}}_{\Lambda \text { nev }}^{(1)}-\tilde{\mathcal{A}}_{\Lambda \text { divev }}^{(1)} \\
& -\left(\tilde{S}_{\Lambda K \mathrm{ev}}, \tilde{\chi}\right),
\end{aligned}
$$

calculated with the action (5.13). We have switched back to the tilde parametrization, used (4.5), and replaced $\left(\tilde{S}_{\Lambda}, \tilde{\chi}\right)$ by $\left(\tilde{S}_{K}+\tilde{S}_{\Lambda K \text { ev }}, \tilde{\chi}\right)$ and $\left(\tilde{S}_{K}, \tilde{\chi}\right)$ by $\tilde{\mathcal{A}}_{\Lambda \text { nev }}^{(1)}$. Both $\mathcal{E}$ and $\hat{\mathcal{A}}_{\Lambda}^{\prime}$ have the structure (3.11) and $\left(\tilde{S}_{\Lambda}, \tilde{S}_{\Lambda}\right)$ is formally evanescent. To fix the notation, let us start from formula (2.20), applied to the $\ell$-loop diagrams containing one $\left(\tilde{S}_{\Lambda}, \tilde{S}_{\Lambda}\right)$ insertion. We write them as sums of contributions of the form

$$
\begin{aligned}
& \mathcal{G}_{\mathcal{A}}^{(\ell)}=\int \tilde{\Phi}^{\alpha_{1}}\left(k_{1}\right) \cdots \tilde{\Phi}^{\alpha_{n}}\left(k_{n}\right) \tilde{K}_{\beta_{1}}\left(k_{n+1}\right) \cdots \\
& \tilde{K}_{\beta_{r}}\left(k_{n+r}\right) T_{\mathcal{A} \mu_{1} \cdots \mu_{p} \alpha_{1} \cdots \alpha_{n}}^{(\ell) \beta_{1} \cdots \beta_{r}} G_{\mathcal{A} \mu_{1} \cdots \mu_{p}}^{(\ell)}\left(k_{1}, \ldots, k_{n+r}\right),
\end{aligned}
$$

where the tensors $T_{\mathcal{A} \mu_{1} \cdots \mu_{p} \alpha_{1} \cdots \alpha_{n}}^{(\ell) \beta_{1} \cdots \beta_{r}}$ are constant and evanescent, and the integrations over momenta are understood. We recall that $G_{\mathcal{A}}^{(\ell) \mu_{1} \cdots \mu_{p}}\left(k_{1}, \ldots, k_{n+r}\right)$ are the integrals coming from Feynman diagrams, once all tensors $\eta_{\mu \nu}, \varepsilon_{\mu \nu \rho \sigma}, \delta_{\hat{\mu} \hat{v}}$, the $\gamma$ matrices, the structure constants $f^{a b c}$ and the matrices $T^{a}$ are moved outside into the structures $T_{\mathcal{A}}^{(\ell)}$. We call the divergent parts of $G_{\mathcal{A}}^{(\ell) \mu_{1} \cdots \mu_{p}}$ "nontrivial" if they are not killed by the structures $T_{\mathcal{A}}^{(\ell)}$.

Let us first reconsider the case $\ell=1$. It is useful to describe the right-hand side of (6.2) from the point of view of the integrals $G_{\mathcal{A}}^{(1) \mu_{1} \cdots \mu_{p}}$. The divergent parts of $G_{\mathcal{A}}^{(1) \mu_{1} \cdots \mu_{p}}$ can be of three types: (a) divergences that are turned into nonevanescent contributions by $T_{\mathcal{A}}^{(1)}$, which are subtracted by $\tilde{\mathcal{A}}_{\Lambda \text { nev }}^{(1)}$; (b) divergences that remain divergent when $T_{\mathcal{A}}^{(1)}$ is applied to them, which are subtracted by $\tilde{\mathcal{A}}_{\Lambda \text { divev }}^{(1)} ;$ (c) divergences that are turned into evanescences by $T_{\mathcal{A}}^{(1)}$, which may be subtracted by further, one-loop, local evanescent terms $\tilde{L}_{\mathrm{ev}}^{(1)}$ with the $\tilde{g}$ structure (3.11). We write $\mathcal{E}=\mathcal{E}_{1}+\mathcal{E}_{2}$, where

$$
\begin{aligned}
& \mathcal{E}_{1}=\left(\tilde{S}_{\Lambda}, \tilde{S}_{\Lambda}\right)-\tilde{\mathcal{A}}_{\Lambda \mathrm{nev}}^{(1)}-\tilde{\mathcal{A}}_{\Lambda \mathrm{divev}}^{(1)}-\tilde{L}_{\mathrm{ev}}^{(1)} \\
& \mathcal{E}_{2}=\tilde{L}_{\mathrm{ev}}^{(1)}-\left(\tilde{S}_{\Lambda K \mathrm{ev}}, \tilde{\chi}\right)
\end{aligned}
$$

The subtractions included in $\mathcal{E}_{1}$ cancel all nontrivial divergences of $G_{\mathcal{A}}^{(1) \mu_{1} \cdots \mu_{p}}$. Instead, $\left\langle\mathcal{E}_{2}\right\rangle$ collects the diagrams with one $\mathcal{E}_{2}$ insertion. They can also be expressed in the form (6.3) and studied along the same lines. From now on we understand that the expressions (6.3) refer to the diagrams with one $\left(\tilde{S}_{\Lambda}, \tilde{S}_{\Lambda}\right)$ insertion or one $\mathcal{E}_{2}$ insertion.

Each contribution $\mathcal{G}_{\mathcal{A}}^{(1)}$ is then equipped with counterterms $\mathcal{G}_{\mathcal{A} \text { counter }}^{(1)}$, so that the difference

$$
\begin{aligned}
\mathcal{G}_{\mathcal{A}}^{(1)}-\mathcal{G}_{\mathcal{A} \text { counter }}^{(1)}= & \int \tilde{\Phi}^{\alpha_{1}}\left(k_{1}\right) \cdots \tilde{\Phi}^{\alpha_{n}}\left(k_{n}\right) \tilde{K}_{\beta_{1}}\left(k_{n+1}\right) \cdots \\
& \tilde{K}_{\beta_{r}}\left(k_{n+r}\right) T_{\mathcal{A} \mu_{1} \cdots \mu_{p} \alpha_{1} \cdots \alpha_{n}}^{(1) \beta_{1} \cdots \beta_{r}} G_{\mathcal{A} \text { subtr }}^{(1) \mu_{1} \cdots \mu_{p}}(k)
\end{aligned}
$$

involves fully convergent subtracted integrals $G_{\mathcal{A} \text { subtr }}^{(1) \mu_{1} \cdots \mu_{p}}$. Now, the evanescences provided by $T_{\mathcal{A}}^{(1)}$ cannot simplify any divergences, so the final result $\hat{\mathcal{A}}_{\Lambda}^{\prime(1)}$ is evanescent, in agreement with (6.1).

At higher loops it is useful to make a similar analysis. We begin with $\ell=2$. The integrals $G_{\mathcal{A}}^{(2) \mu_{1} \cdots \mu_{p}}$ are automatically equipped with the counterterms that subtract their nontrivial subdivergences: first, the action $\hat{S}_{\Lambda}^{\prime}$ is equipped 
with its own counterterms and, second, the subtractions contained in $\mathcal{E}_{1}$ provide counterterms for the integrals $G_{\mathcal{A}}^{(1) \mu_{1} \cdots \mu_{p}}$ associated with $\left(\tilde{S}_{\Lambda}, \tilde{S}_{\Lambda}\right)$. Instead, the two-loop contributions of $\mathcal{E}_{2}$ do not have subdivergences, because $\mathcal{E}_{2}$ is oneloop. When we include counterterms for subdivergences, we can identify subtracted integrals $G_{\mathcal{A}}^{(2) \mu_{1} \cdots \mu_{p}}-G_{\mathcal{A} \text { subdiv }}^{(2) \mu_{1} \cdots \mu_{p}}$ that have local divergent parts $G_{\mathcal{A} \text { div }}^{(2) \cdots \mu_{p}}$ (by the theorem of locality of counterterms) and possibly nonlocal finite parts $G_{\mathcal{A} \text { finite }}^{(2) \mu_{1} \cdots \mu_{p}}$. When $T_{\mathcal{A}}^{(2)}$ acts on $G_{\mathcal{A} \text { div }}^{(2) \mu_{1} \cdots \mu_{p}}$, it gives local contributions to $\hat{\mathcal{A}}_{\Lambda}^{\prime(2)}$, which can be nonevanescent (due to simplified divergences), evanescent or still divergent. However, local contributions must have the structure (3.11), which implies that they are zero. Indeed, using the tilde parametrization, they are polynomial in the dimensionful parameters of $\tilde{S}_{\Lambda}$ and carry an overall factor $\tilde{g}^{2}$, which has dimension 16 . We conclude that the overall divergences $G_{\mathcal{A} \text { div }}^{(2) \mu_{1} \cdots \mu_{p}}$ are trivial, because they are killed by $T_{\mathcal{A}}^{(2)}$. When $T_{\mathcal{A}}^{(2)}$ acts on $G_{\mathcal{A} \text { finite }}^{(2) \mu_{1} \cdots \mu_{p}}$ it just gives (possibly nonlocal) evanescent contributions to $\hat{\mathcal{A}}_{\Lambda}^{\prime(2)}$. Finally, we have

$\hat{\mathcal{A}}_{\Lambda}^{\prime(2)}=\mathcal{O}(\varepsilon)$.

Therefore, (6.1) is promoted to the next order, and we can write $\hat{\mathcal{A}}_{\Lambda}^{\prime}=\mathcal{O}(\varepsilon)+\mathcal{O}\left(\hbar^{3}\right)$, where now " $\mathcal{O}(\varepsilon)$ " includes the evanescent contributions appearing on the right-hand side of (6.4).

At this point we can proceed by induction. Assume that for some $\ell \geqslant 2$,

$\hat{\mathcal{A}}_{\Lambda}^{\prime}=\mathcal{O}(\varepsilon)+\mathcal{O}\left(\hbar^{\ell+1}\right)$,

and that the overall divergent parts $G_{\mathcal{A} \text { div }}^{(L) \mu_{1} \cdots \mu_{p}}$ of the subtracted integrals $G_{\mathcal{A}}^{(L) \mu_{1} \cdots \mu_{p}}-G_{\mathcal{A} \text { subdiv }}^{(L) \mu_{1} \cdots \mu_{p}}$ are trivial for $2 \leqslant L \leqslant \ell$. Denote the contributions of order $\hbar^{\ell+1}$ to $\hat{\mathcal{A}}_{\Lambda}^{\prime}$ with $\hat{\mathcal{A}}_{\Lambda}^{\prime(\ell+1)}$. A diagrammatic analysis similar to the one carried out above shows that $\hat{\mathcal{A}}_{\Lambda}^{\prime(\ell+1)}$ is the sum of a local part $\hat{\mathcal{A}}_{\Lambda \text { loc }}^{\prime(\ell+1)}=\sum T_{\mathcal{A}}^{(\ell+1)} G_{\mathcal{A} \text { div }}^{(\ell+1)}$, plus a possibly nonlocal evanescent part $\hat{\mathcal{A}}_{\Lambda \mathrm{ev}}^{(\ell+1)}=\sum T_{\mathcal{A}}^{(\ell+1)} G_{\mathcal{A} \text { finite }}^{(\ell+1)}$. However, $\hat{\mathcal{A}}_{\Lambda \text { loc }}^{(\ell+1)}$ must have the structure (3.11), which means that it vanishes. In the end, $G_{\mathcal{A d i v}}^{(\ell+1) \mu_{1} \cdots \mu_{p}}$ are also trivial, and $\hat{\mathcal{A}}_{\Lambda}^{\prime(\ell+1)}=\hat{\mathcal{A}}_{\Lambda \text { ev }}^{(\ell+1)}$. Thus, if the inductive assumptions hold for some $\ell$, they must also hold with $\ell \rightarrow \ell+1$ and therefore for $\ell=\infty$. We conclude that the anomaly is evanescent to all orders:

$\hat{\mathcal{A}}_{\Lambda}^{\prime}=\left(\hat{\Gamma}_{\Lambda}^{\prime}, \hat{\Gamma}_{\Lambda}^{\prime}\right)=\mathcal{O}(\varepsilon)$.

This result proves that if the final theory is anomaly-free at one loop, the higher-derivative theory $S_{\Lambda}$ is anomalyfree to all orders. It is important to stress that the DHDregularization framework provides the subtraction scheme where this property is manifest: after the subtraction of $\left(S_{K}, \chi\right)$ at one loop, no analogous subtractions are necessary at higher orders.

This is not the final result we want, though. To get there we still need to take $\Lambda$ to infinity and complete the DHD limit.

\section{Manifest Adler-Bardeen theorem in the final theory}

We are finally ready to study anomaly cancellation to all orders in the final theory. In this section we study the $\Lambda$ dependence and complete the DHD limit, according to the rules of Sect. 3.1. The subtraction of $\Lambda$ divergences proceeds relatively smoothly, and preserves the master equation to all orders up to terms that vanish in the DHD limit.

Call $S_{n}$ and $\Gamma_{n}$ the action and the $\Gamma$ functional DHDrenormalized up to (and including) $n$ loops, where $S_{0}=$ $\hat{S}_{\Lambda}^{\prime}=\hat{S}_{\Lambda}-\chi / 2$ is the action (5.13). The action $S_{n}$ must satisfy two inductive assumptions to all orders in $\hbar$ :

(I) $\Gamma_{n}$ has a regular limit for $\varepsilon \rightarrow 0$ at fixed $\Lambda$, and

(II) the local functional

$\left(S_{n}, S_{n}\right) \equiv \mathcal{E}_{n}$

is "truly $\varepsilon$-evanescent at fixed $\Lambda$ ", that is to say a local functional such that $\left\langle\mathcal{E}_{n}\right\rangle$ tends to zero when $\varepsilon \rightarrow 0$ at fixed $\Lambda$.

More precisely, $\Gamma_{n}$ is a sum of terms (3.14) up to $n$ loops (because it is DHD-convergent to that order) and a sum of terms (3.12) from $n+1$ loops onwards. Instead, $\left\langle\mathcal{E}_{n}\right\rangle=$ $\left(\Gamma_{n}, \Gamma_{n}\right)$ contains the terms (3.14) except $\varepsilon^{0} \Lambda^{0}$ and $\varepsilon^{0} / \Lambda$ up to $n$ loops, and the terms (3.12) except $\varepsilon^{0}$ from $n+1$ loops onwards. Thanks to (6.6) we know that the inductive hypotheses are true for $n=0$.

The theorem of locality of counterterms ensures that the $(n+1)$-loop divergent part $\Gamma_{n \text { div }}^{(n+1)}$ of $\Gamma_{n}$ is a local functional. Since $\Gamma_{n}$ has a regular limit for $\varepsilon \rightarrow 0$ at fixed $\Lambda, \Gamma_{n \operatorname{div}}^{(n+1)}$ contains only divergences in $\Lambda$, not in $\varepsilon$. Precisely, we can write

$\Gamma_{n \operatorname{div}}^{(n+1)}=\Gamma_{n \text { divnev }}^{(n+1)}+\Gamma_{n \text { divfev }}^{(n+1)}$,

where $\Gamma_{n \text { divnev }}^{(n+1)}$ and $\Gamma_{n \text { divfev }}^{(n+1)}$ collect the terms $\varepsilon^{0} \Lambda$ and $\hat{\delta} \varepsilon^{0} \Lambda$ of the list (3.13), respectively.

Now we study the $(n+1)$-loop divergent part of $\left(\Gamma_{n}, \Gamma_{n}\right)$. We take the $(n+1)$-loop DHD-divergent non- $\varepsilon$-evanescent part of

$\left(\Gamma_{n}, \Gamma_{n}\right)=\left\langle\left(S_{n}, S_{n}\right)\right\rangle=\left\langle\mathcal{E}_{n}\right\rangle$, 
which means the terms of types $\varepsilon^{0} \Lambda$ of the list (3.13). Recall that $S_{\Lambda}$ is equal to the action $S_{r}$ of $(2.15)$ plus $\mathcal{O}\left(1 / \Lambda^{6}\right)$ terms, so $\left(S_{\Lambda}-S_{r}, \Gamma_{n \text { div }}^{(n+1)}\right)$ is convergent for $\Lambda \rightarrow \infty$. Moreover, $S_{r}$ is equal to $S_{\mathrm{gf}}$, which by (2.3) is non- $\varepsilon$-evanescent, plus $\int \bar{\psi}_{R}^{I} \iota \not \partial \psi_{R}^{I}$ plus $\varepsilon$-evanescent terms. Noting that the divergent part of $\left\langle\mathcal{E}_{n}\right\rangle$ is just made of terms $\hat{\delta} \varepsilon^{0} \Lambda$, we obtain

$\left(S_{\text {gf }}, \Gamma_{n \text { divnev }}^{(n+1)}\right)=0$.

Deriving (7.3) from (7.2) we have expanded $\Gamma_{n}=$ $\sum_{k=0}^{\infty} \hbar^{k} \Gamma_{n}^{(k)}$ in powers of $\hbar$ and dropped all contributions $\left(\Gamma_{n}^{(k)}, \Gamma_{n}^{(n+1-k)}\right)$ with $0<k<n+1$, because they are convergent in the DHD limit. Note that $\Gamma_{n}^{(k)}, 0<k<n+1$, may contain terms $\varepsilon \Lambda$. Now, the powers of $\Lambda$ can get simplified inside $\left(\Gamma_{n}^{(k)}, \Gamma_{n}^{(n+1-k)}\right)$. However, $\Gamma_{n}$ is convergent for $\varepsilon \rightarrow 0$ and the antiparentheses cannot generate poles, so the resulting contributions remain negligible in the DHD limit. We must just pay attention not to manipulate the terms $\varepsilon \Lambda$ in inconvenient ways (see Sect. 3.1 for details).

Since the theory is power-counting renormalizable, (7.3) is another little cohomological problem, therefore it can be solved directly. Moreover, it is a purely four-dimensional problem, since all $\varepsilon$-evanescent terms have been dropped. Its solution is well known and states that $\Gamma_{n \text { divnev }}^{(n+1)}$ may be reabsorbed by redefining the parameters of $S_{\mathrm{gf}}$ and making a canonical transformation inside $S_{\mathrm{gf}}$. Using the nonrenormalization of the $B$ - and $K_{\bar{C}}$-dependent terms, and power counting, the canonical transformation is generated by a functional

$$
\begin{aligned}
F_{n}\left(\Phi, K^{\prime}\right)= & \int \sum_{i}\left(Z_{n A i}^{1 / 2} A_{\mu}^{a_{i}} K^{\prime \mu a_{i}}+Z_{n C i}^{1 / 2} C^{a_{i}} K_{C}^{\prime a_{i}}\right. \\
& \left.+Z_{n A i}^{-1 / 2} \bar{C}^{a_{i}} K_{\bar{C}}^{\prime a_{i}}+Z_{n A i}^{-1 / 2} B^{a_{i}} K_{B}^{\prime a_{i}}\right) \\
& +\int\left(\bar{\psi}_{L}^{I} Z_{n J I}^{1 / 2 *} K_{\psi}^{\prime J}+\bar{K}_{\psi}^{\prime I} Z_{n I J}^{1 / 2} \psi_{L}^{J}\right),
\end{aligned}
$$

and the parameter redefinitions read

$r_{i}^{\prime}=Z_{n i} r_{i}, \quad \xi_{i}^{\prime}=\xi_{i} Z_{n A i}$,

where $Z_{n A i}, Z_{n C i}, Z_{n I J}$, and $Z_{n i}$ are $\varepsilon$-independent $\Lambda$ divergent renormalization constants. The $r_{i}$ redefinitions encode the renormalizations of gauge couplings. Instead, the $\xi_{i}$ redefinitions follow from the nonrenormalization of the terms quadratic in $B$. In the parametrization we are using there are no redefinitions of $g$ and $\zeta_{i}$.

Making the canonical transformation (7.4) and the redefinitions (7.5) on $S_{\text {gf we get }}$

$S_{\mathrm{gf}} \rightarrow S_{\mathrm{gf}}-\Gamma_{n \text { div nev }}^{(n+1)}+\mathcal{O}\left(\hbar^{n+2}\right)$.

However, the classical action we have been using is not $S_{\mathrm{gf}}$, and not even $S_{r}=S_{\mathrm{gf}}+S_{\mathrm{LR}}+S_{\mathrm{ev}}$, but $S_{n}$, whose classical limit is $S_{\Lambda}$, therefore we must inquire what happens by making the operations (7.4) and (7.5) on $S_{\Lambda}$.
Let us begin from $S_{r}$. Since $S_{\mathrm{LR}}$ is nonrenormalized, we must also make the redefinitions

$\varsigma_{I J}^{\prime}=\varsigma_{I K} Z_{n K J}^{-1 / 2}$.

When we apply (7.4) and (7.5) to $S_{\mathrm{ev}}$ we generate new formally $\varepsilon$-evanescent, $\Lambda$-divergent terms of order $\hbar^{n+1}$, which change $\Gamma_{n \text { divfev }}^{(n+1)}$ into some new $\Gamma_{n \text { divfev }}^{\prime(n+1)}$, plus $\mathcal{O}\left(\hbar^{n+2}\right)$. The divergences $\Gamma_{n \text { divfev }}^{\prime(n+1)}$ are not constrained by gauge invariance, but just locality and power counting. They can be subtracted by redefining the parameters $\eta$ of $S_{\mathrm{ev}}$, since $S_{\mathrm{ev}}$ was added precisely for this purpose.

We denote the operations that subtract $\Gamma_{n \text { div }}^{(n+1)}$ with $T_{n}$. They include the canonical transformation (7.4), the redefinitions (7.5) and (7.6), and the $\eta$ redefinitions that subtract $\Gamma_{n \text { divfev }}^{\prime(n+1)}$. Note that $T_{n}=1+\mathcal{O}\left(\hbar^{n+1}\right)$. We have

$T_{n} S_{r}=S_{r}-\Gamma_{n \operatorname{div}}^{(n+1)}+\mathcal{O}\left(\hbar^{n+2}\right)$.

It remains to check what happens when the operations $T_{n}$ act on $S_{\Lambda}$. Observe that, since no $\varepsilon$ divergences are around, the operations $T_{n}$ are independent of $\varepsilon$ and divergent in $\Lambda$. However, the difference $S_{\Lambda}-S_{r}$ is of order $1 / \Lambda^{6}$ and the operations $T_{n}$ cannot contain powers of $\Lambda$ greater than 4 . Thus, $\left(T_{n}-1\right)\left(S_{\Lambda}-S_{r}\right)$ vanishes in the DHD limit. Call $S_{n+1}$ the action obtained by applying $T_{n}$ on $S_{n}$. We have

$$
\begin{aligned}
S_{n+1}= & T_{n} S_{n}=S_{n}+\left(T_{n}-1\right) S_{\Lambda}+\mathcal{O}\left(\hbar^{n+2}\right)=S_{n} \\
& +\left(T_{n}-1\right) S_{r}+\left(T_{n}-1\right)\left(S_{\Lambda}-S_{r}\right)+\mathcal{O}\left(\hbar^{n+2}\right) \\
= & S_{n}-\Gamma_{n \operatorname{div}}^{(n+1)}+\left(T_{n}-1\right)\left(S_{\Lambda}-S_{r}\right)+\mathcal{O}\left(\hbar^{n+2}\right) .
\end{aligned}
$$

This formula tells us that the operations $T_{n}$ do renormalize the divergences due to $S_{n}$ in the DHD limit. Therefore, $S_{n+1}$ is the $(n+1)$-loop DHD-renormalized action, namely it gives a generating functional $\Gamma_{n+1}$ that is convergent up to (and including) $n+1$ loops in the DHD limit.

Moreover, since the canonical transformations generated by (7.4) act multiplicatively on fields and sources, the operations $T_{n}$ act on the $\Gamma$ functional precisely as they act on the action. Therefore, $\Gamma_{n+1}=T_{n} \Gamma_{n}$. Since the operations $T_{n}$ are $\varepsilon$-independent, we conclude that $\Gamma_{n+1}$ is regular when $\varepsilon \rightarrow 0$ at fixed $\Lambda$, to all orders in $\hbar$, which promotes the inductive assumption (I) to $n+1$ loops.

Finally, the operations $T_{n}$ preserve the antiparentheses. Applying them to (7.1) we also obtain

$\left(S_{n+1}, S_{n+1}\right)=T_{n} \mathcal{E}_{n}$.

Now, taking the average of this equation we get

$$
\begin{aligned}
T_{n}\left\langle\mathcal{E}_{n}\right\rangle_{n} & =T_{n}\left(\Gamma_{n}, \Gamma_{n}\right)=\left(\Gamma_{n+1}, \Gamma_{n+1}\right) \\
& =\left\langle\left(S_{n+1}, S_{n+1}\right)\right\rangle_{n+1}=\left\langle T_{n} \mathcal{E}_{n}\right\rangle_{n+1},
\end{aligned}
$$

where $\langle\cdots\rangle_{k}$ means that the average is calculated with the action $S_{k}$. If we take the limit of $T_{n}\left\langle\mathcal{E}_{n}\right\rangle_{n}$ for $\varepsilon \rightarrow 0$ at fixed $\Lambda$ we get zero, because by assumption (II) $\left\langle\mathcal{E}_{n}\right\rangle_{n}$ tends to zero 
for $\varepsilon \rightarrow 0$ at fixed $\Lambda$. We conclude that the local functional $\mathcal{E}_{n+1} \equiv T_{n} \mathcal{E}_{n}$ is truly $\varepsilon$ evanescent at fixed $\Lambda$. Therefore, assumption (II) is also promoted to $n+1$ loops.

Since all inductive assumptions have been successfully promoted to $n+1$ loops, the DHD-renormalized action $S_{R}=$ $S_{\infty}$ satisfies

$\left(S_{R}, S_{R}\right)=\mathcal{E}_{R}$,

where $\left\langle\mathcal{E}_{R}\right\rangle$ vanishes in the DHD limit, because it contains only the terms of (3.14) except $\varepsilon^{0} \Lambda^{0}$ and $\varepsilon^{0} / \Lambda$. Finally, the DHD-renormalized $\Gamma$ functional $\Gamma_{R}=\Gamma_{\infty}$ is such that the anomaly functional

$\mathcal{A}_{R}=\left(\Gamma_{R}, \Gamma_{R}\right)=\left\langle\left(S_{R}, S_{R}\right)\right\rangle_{S_{R}}=\left\langle\mathcal{E}_{R}\right\rangle=\mathcal{O}(\varepsilon)$

tends to zero in the DHD limit, which means that gauge anomalies cancel out to all orders.

The DHD framework defines a subtraction scheme where the cancellation takes place naturally and manifestly. In any other framework, the right scheme must be identified stepby-step, from two loops onwards, by fine-tuning local counterterms.

Some final comments are in order. Because of (4.7) higherorder divergent terms of the form $\Lambda^{p} \ln ^{k} \Lambda / \varepsilon$ are generated along the way. They appear in $S_{R}$ and in the partially renormalized actions $S_{n}$. Our renormalization procedure (which is just made of redefinitions of parameters, fields, and sources) makes them cancel opposite contributions coming from diagrams. Therefore, they do not appear in the $\Gamma$ functionals $\Gamma_{R}$ and $\Gamma_{n}$, which are indeed regular in the limit $\varepsilon \rightarrow 0$ at $\Lambda$ fixed.

In several steps of the proof we have used the fact that $S_{\Lambda}=S_{r}+\mathcal{O}\left(1 / \Lambda^{6}\right)$. It is important that the higher-derivative regularized classical action $S_{\Lambda}$ does not contain terms with fewer inverse powers of $\Lambda$. Consistently with this, renormalization does not require to turn them on. The operations $T_{n}$ may contain power-like divergences, which can generate terms with less than six inverse powers of $\Lambda$ when they act on $S_{\Lambda}-S_{r}$. Those terms are at least one loop and not divergent, so they do not affect the structure of the classical action $S_{\Lambda}$.

\section{Standard Model and more general theories}

In this section we show how to extend the proof of the previous sections to the standard model and the most general perturbatively unitary, power-counting renormalizable theories. We just need to include photons $V_{\mu}$, scalar fields $\varphi$, and right-handed fermions $\chi_{R}$, which were dropped so far for simplicity. Depending on the representations, we can also add Majorana masses to the fermions $\psi_{L}$.

We begin from the fermions. The starting classical action (2.1) is modified as follows:

$S_{c} \rightarrow S_{c}+\int \bar{\chi}_{R}^{I} l \not D \chi_{R}^{I}+S_{m}$, where $S_{m}$ collects the mass terms, when allowed by the representations:

$$
\begin{aligned}
S_{m}= & -\int\left(\bar{\chi}_{R}^{I} m_{I J} \psi_{L}^{J}+\bar{\psi}_{L}^{I} m_{J I}^{*} \chi_{R}^{J}\right)-\int\left(\bar{\psi}_{L}^{c I} M_{I J} \psi_{L}^{J}\right. \\
& \left.+\bar{\psi}_{L}^{I} M_{J I}^{*} \psi_{L}^{c J}\right)-\int\left(\bar{\chi}_{R}^{c I} M_{I J}^{\prime} \chi_{R}^{J}+\bar{\chi}_{R}^{I} M_{J I}^{* \prime} \chi_{R}^{c J}\right) .
\end{aligned}
$$

The functional $S_{K}$ that collects the symmetry transformations is also extended:

$S_{K} \rightarrow S_{K}+g \int\left(\bar{\chi}_{R}^{I} T^{a} C^{a} K_{\chi}^{I}+\bar{K}_{\chi}^{I} T^{a} C^{a} \chi_{R}^{I}\right)$.

Clearly, $\Psi$ and $\left(S_{K}, \Psi\right)$ are unmodified. To regularize the right-handed fermions we mirror what we did for the lefthanded ones. In the same way as we added partners $\psi_{R}$ for $\psi_{L}$ that decouple in four dimensions, we add partners $\chi_{L}$ for $\chi_{R}$ that also decouple when $D \rightarrow 4$. The correction to $S_{\mathrm{LR}}$ is

$$
\begin{aligned}
S_{\mathrm{LR}} \rightarrow & S_{\mathrm{LR}}+\varsigma_{I J}^{\prime} \int \bar{\chi}_{L}^{I} \iota \not \partial \chi_{R}^{J}+\varsigma_{J I}^{*} \int \bar{\chi}_{R}^{I} \iota \not \partial \chi_{L}^{J} \\
& +\int \bar{\chi}_{L}^{I} \iota \not \partial \chi_{L}^{I} .
\end{aligned}
$$

Massive terms involving the regularizing partners $\psi_{R}^{I}$ and $\chi_{L}^{I}$ can also be added. Differently from (8.1), they are not renormalized, so their coefficients must be independent of the ones appearing in (8.1). The evanescent corrections $S_{\mathrm{ev}}$ of (2.16) are affected only in the sector $S_{c e v}$, which is extended to include terms such as the integrals of

$\bar{\chi}_{R}^{I} \imath \not \partial \psi_{L}^{J}, \quad \bar{\psi}_{L}^{I} \iota \not \partial \chi_{R}^{J}, \quad \bar{\chi}_{R}^{I} A \psi_{L}^{J}, \quad \bar{\psi}_{L}^{I} A \chi_{R}^{J}$,

multiplied by independent constants. Evanescent terms of the Majorana type may also be allowed.

Next, we add the higher-derivative regularizing terms

$\int \bar{\chi}_{R}^{I} \iota \not D\left[\left(\frac{D^{2}}{\Lambda^{2}}\right)^{3}-\left(\frac{\partial^{2}}{\Lambda^{2}}\right)^{3}\right] \chi_{R}^{I}+\int \bar{\chi}^{I} \iota \not \partial\left(\frac{\partial^{2}}{\Lambda^{2}}\right)^{3} \chi^{I}$

to $S_{\Lambda}$, where $\chi^{I}=\chi_{L}^{I}+\chi_{R}^{I}$. The gauge fermion $\Psi_{\Lambda}$ does not change, as well as $S_{\Lambda \text { ev }}-S_{c e v}$. Tilde fields and sources are defined as before and every argument of the proof can be extended straightforwardly. Now, wave-function renormalization constants can mix right-handed fermions with conjugates of left-handed ones. The contributions of right-handed fermions to the one-loop anomalies $\mathcal{A}_{f \text { nev }}^{(1)}=\mathcal{A}_{\Lambda \text { nev }}^{(1)}$ are given by a formula similar to (5.11), the only difference being that the trace appearing in the Bardeen term on the righthand side is calculated on the appropriate representations $T_{R}^{a}$ $\left(C \rightarrow C^{a} T_{R}^{a}, A_{\mu} \rightarrow A_{\mu}^{a} T_{R}^{a}\right)$ and is multiplied by a further minus sign. The one-loop gauge anomalies $\mathcal{A}_{f \text { nev }}^{(1)}$ are trivial when the Bardeen terms cancel out in the total, and there exists a local functional $\chi(g A)$ such that $\mathcal{A}_{f \text { nev }}^{(1)}=\left(S_{K}, \chi\right)$. 
Scalars may be added by making the replacements

$$
\begin{aligned}
S_{c} \rightarrow & S_{c}+\int\left(D_{\mu} \varphi\right)^{\dagger}\left(D^{\mu} \varphi\right)+m^{2} \int \varphi^{\dagger} \varphi \\
& +\frac{\lambda}{4} \int\left(\varphi^{\dagger} \varphi\right)^{2}+S_{Y}, \\
S_{K} \rightarrow & S_{K}-g \int\left(\varphi^{\dagger} T^{a} C^{a} K_{\varphi}+K_{\varphi}^{\dagger} T^{a} C^{a} \varphi\right),
\end{aligned}
$$

where $S_{Y}$ denotes the Yukawa terms. As before, the renormalized action is linear in the sources $K$, by ghost number conservation and power counting. The evanescent corrections $S_{c \text { ev }}$ include new terms such as the integrals of

$$
\left(\partial_{\hat{\mu}} \varphi\right)^{\dagger}\left(\partial^{\hat{\mu}} \varphi\right), \quad\left(\partial_{\hat{\mu}} \varphi\right)^{\dagger} T^{a}\left(A^{\hat{\mu} a} \varphi\right),
$$

while $S_{\Lambda \text { ev }}-S_{c \text { ev }}$ does not change. The higher-derivative regularizing terms are

$\int\left(D_{\mu} \varphi\right)^{\dagger}\left(\frac{D^{2}}{\Lambda^{2}}\right)^{4}\left(D^{\mu} \varphi\right)$,

so the tilde fields and sources

$\tilde{\varphi}=\frac{\varphi}{\Lambda^{4}}, \quad \tilde{K}_{\varphi}=\Lambda^{4} K_{\varphi}$,

are such that $[\tilde{g} \tilde{\varphi}]=5,\left[\tilde{g} \tilde{K}_{\varphi}\right]=14$. With these choices, the matter fields and their sources still cannot contribute to the one-loop counterterms $\tilde{\Gamma}_{\Lambda \text { div }}^{(1)}$ of the higher-derivative theory $\tilde{S}_{\Lambda}$, nor to the nonevanescent one-loop gauge anomalies $\tilde{\mathcal{A}}_{\Lambda \text { nev }}^{(1)}$. Moreover, we still have $S_{\Lambda}-S_{r}=\mathcal{O}\left(1 / \Lambda^{6}\right)$. Therefore, all arguments used in the proof of the previous sections generalize straightforwardly.

Finally, we add photons. Assume that the group $G$ contains $N U$ (1) factors and denote their gauge fields with $V_{\mu}^{u}, u=$ $1, \ldots N$. Then make the replacements

$$
\begin{aligned}
S_{c} \rightarrow & S_{c}-\frac{1}{4} \int \zeta_{u v} W_{\mu \nu}^{u} W^{v \mu \nu}, \\
& D_{\mu} \pi^{I} \rightarrow D_{\mu} \pi^{I}+i Q^{u} V_{\mu}^{u} \pi^{I}, \\
S_{K} \rightarrow & S_{K}-\int\left(\partial_{\mu} C^{u}\right) K^{\mu u} \\
& -\imath g \int C^{u} \sum_{\pi}\left(\pi^{I \dagger} Q K_{\pi}^{I}-K_{\pi}^{I \dagger} Q \pi^{I}\right),
\end{aligned}
$$

where $W_{\mu \nu}^{u}=\partial_{\mu} V_{v}^{u}-\partial_{\nu} V_{\mu}^{u}, \zeta_{u v}$ is an invertible constant matrix, $\pi^{I}$ is any matter field in the irreducible representation $R^{I}$ of $G$, and $\pi^{I \dagger}, K_{\pi}^{I \dagger}$ stand for $\bar{\pi}^{I}, \bar{K}_{\pi}^{I}$ if $\pi^{I}$ is a fermion. We define extended $G$ indices $\hat{a}, \hat{b}, \ldots$ to include both sets of indices $u, v, \ldots$ and $a, b, \ldots$, and write $A_{\mu}^{\hat{a}}=\left\{V_{\mu}^{u}, A_{\mu}^{a}\right\}$. The $U(1)$ charges of matter fields are denoted by $g q_{I}^{u}$. We also write $T^{\hat{a}}=\left\{i Q^{u}, T^{a}\right\}$, where $Q^{u}$ acts on $\pi^{I}$ by multiplying it by $q_{I}^{u}$. The change of the gauge fermion (2.4) is

$\Psi(\Phi) \rightarrow \Psi(\Phi)+\int \bar{C}^{u}\left(\partial^{\mu} A_{\mu}^{u}+\frac{\xi_{u v}}{2} B^{v}\right)$.
The sector $S_{c \text { ev }}$ of $S_{\text {ev }}$ is also extended, to include $V$ dependent evanescent terms similar to those already met in (2.19), (8.2), and (8.3). Instead, $S_{\Lambda \mathrm{ev}}-S_{c \mathrm{cev}}$ remains the same, since the $U(1)$ ghosts decouple.

The action $S_{c \Lambda}$ is extended to include the higher-derivative regularizing terms

$-\frac{1}{4} \int W_{\mu \nu}^{u}\left(\frac{\partial^{2}}{\Lambda^{2}}\right)^{8} W^{u \mu \nu}$,

while the change of the gauge fermion is

$\Psi_{\Lambda}(\Phi) \rightarrow \Psi_{\Lambda}(\Phi)+\int \bar{C}^{u}\left(Q(\square) \partial^{\mu} V_{\mu}^{u}+\frac{1}{2} P_{u v}(\square) B^{v}\right)$,

where

$P_{u v}(\square)=\xi_{u v}+\frac{\delta_{u v} \xi^{\prime}}{\Lambda^{16}} \square^{8}$.

Finally, $S_{\Lambda \mathrm{ev}}$ inherits the modifications made on $S_{c \mathrm{ev}}$. Tilde fields and sources are defined as before. The one-loop renormalization of the higher-derivative theory $\tilde{S}_{\Lambda}$ is made of the replacements (4.7) plus similar replacements,

$\zeta_{u v} \rightarrow \zeta_{u v}+\frac{f_{u v}}{\varepsilon} g^{2}$

for $\zeta_{u v}$, where $f_{u v}$ are calculable constants.

Let us describe the nontrivial contributions to the one-loop gauge anomalies $\mathcal{A}_{f \text { nev }}^{(1)}$. We have terms of the Badreen type and terms proportional to $C^{u} W_{\mu \nu}^{v} W^{z \mu \nu}$. Using differential forms, the terms of the Bardeen type are linear combinations of $\mathcal{B}_{1}=\int \operatorname{Tr}\left[d C_{\wedge} A \wedge d A\right]$ and $\mathcal{B}_{2}=\int \operatorname{Tr}\left[d C_{\wedge} A \wedge A \wedge A\right]$, as in (5.11), where now $C=C^{\hat{a}} T_{f}^{\hat{a}}, A=A_{\mu}^{\hat{a}} T_{f}^{\hat{a}} d x^{\mu}, d=$ $d x^{\mu} \partial_{\mu}$ and $T_{f}^{\hat{a}}$ are the matrices $T^{\hat{a}}$ restricted to the fermions. The coefficient of $\mathcal{B}_{1}$ is the same as in (5.11), apart from the minus sign associated with right-handed fermions. The coefficient of $\mathcal{B}_{2}$ is uniquely determined by the coefficient of $\mathcal{B}_{1}$, but it differs from the one of (5.11) any time $U(1)$ gauge fields and/or ghosts are involved. The terms proportional to $C W_{\mu \nu} W^{\mu \nu}$ can only appear in (unusual) situations where global $U(1)$ gauge symmetries are potentially anomalous. One-loop gauge anomalies are trivial when all these terms cancel out, and there exists a local functional $\chi(g A)$ such that $\mathcal{A}_{f \text { nev }}^{(1)}=\left(S_{K}, \chi\right)$.

The correction to the canonical transformation (7.4) reads

$$
\begin{aligned}
F_{n}\left(\Phi, K^{\prime}\right) \rightarrow & F_{n}\left(\Phi, K^{\prime}\right)+\int\left(V_{\mu}^{u} Z_{n u v}^{1 / 2} K^{\prime \mu v}+C^{u} Z_{n u v}^{1 / 2} K_{C}^{\prime v}\right. \\
& \left.+\bar{C}^{u} Z_{n u v}^{-1 / 2} K_{\bar{C}}^{\prime v}+B^{u} Z_{n u v}^{-1 / 2} K_{B}^{\prime v}\right),
\end{aligned}
$$

and the redefinitions (7.5) are accompanied by

$q_{I}^{u \prime}=Z_{n u v}^{-1 / 2} q_{I}^{v}, \quad \xi_{u v}^{\prime}=Z_{n u w}^{1 / 2} \xi_{w z} Z_{n z v}^{1 / 2}$,

so that the $U(1)$ gauge-fixing sector $\left(S_{K}, \Psi\right)$, including the ghost action, as well as the $U(1)$ sector of $S_{K}$, are nonrenormalized. 
With the rules of this section gauge anomalies manifestly cancel to all orders in the most general perturbatively unitary, renormalizable gauge theory coupled to matter, as long as they vanish at one loop. We stress again that the proof we have given also works when the theory is conformal or finite, or the first coefficients of its beta functions vanish, where instead RG techniques are powerless.

\section{Conclusions}

We have reconsidered the Adler-Bardeen theorem, focusing on the cancellation of gauge anomalies to all orders, when they are trivial at one loop. The proof we have worked out is more powerful than the ones appeared so far and makes us understand aspects that the previous derivations were unable to clarify. Key ingredients of our approach are the BatalinVilkovisky formalism and a regularization technique that combines the dimensional regularization with the higherderivative gauge invariant regularization. The most important result is the identification of the subtraction scheme where gauge anomalies manifestly cancel to all orders. We have not used renormalization-group arguments, so our results apply to the most general perturbatively unitary, renormalizable gauge theories coupled to matter, including conformal field theories, finite theories, and theories where the first coefficients of the beta functions vanish.

In view of future generalizations to wider classes of quantum field theories, we have paid attention to a considerable amount of details and delicate steps that emerge along with the proof. We are convinced that the techniques developed here may help us identify the right tools to upgrade the formulation of quantum field theory and simplify the proofs of all-order theorems.

Open Access This article is distributed under the terms of the Creative Commons Attribution License which permits any use, distribution, and reproduction in any medium, provided the original author(s) and the source are credited.

Funded by $\mathrm{SCOAP}^{3}$ / License Version CC BY 4.0.

\section{Appendix A: Calculation of one-loop anomalies}

In this appendix we illustrate our approach by calculating the one-loop coefficient of the Bardeen anomaly in chiral gauge theories. That coefficient is scheme independent, so we can work at $\Lambda=\infty$, which means use the dimensionally regularized action $S_{r}$ of (2.15). Actually, we can equivalently use the action $S_{r 0}$ of (2.8), because it is easy to check that the contributions due to $S_{\mathrm{ev}}$ do not contain fermion loops. Therefore, they cannot generate the tensor $\varepsilon^{\mu \nu \rho \sigma}$.

For simplicity, we first work with chiral QED and then generalize the result to non-Abelian theories. The action reads

$$
\begin{aligned}
S_{r 0}(\Phi, K)= & -\frac{1}{4} \int F_{\mu \nu} F^{\mu \nu}+\int \bar{\psi} \iota \gamma^{\mu} \partial_{\mu} \psi \\
& -\int q_{L} \bar{\psi}_{L} \gamma^{\mu} A_{\mu} \psi_{L}+\left(S_{K}, \Psi\right)+S_{K}, \\
S_{K}= & -\int\left(\partial_{\mu} C\right) K^{\mu}+\imath q_{L} \int\left(\bar{\psi}_{L} C K_{\psi}\right. \\
& \left.+\bar{K}_{\psi} C \psi_{L}\right)-\int B K_{\bar{C}},
\end{aligned}
$$

where $q_{L}$ is the charge and the gauge fermion is

$\Psi=\int \bar{C}\left(\partial^{\mu} A_{\mu}+\frac{\xi}{2} B\right)$.

We have

$$
\begin{aligned}
& \left(S_{r 0}, S_{r 0}\right)=-2 q_{L} \int C\left(\bar{\psi}_{L} \hat{\partial} \psi_{R}+\left(\partial_{\hat{\mu}} \bar{\psi}_{R}\right) \gamma^{\hat{\mu}} \psi_{L}\right) \\
& =2 \int C\left(\partial_{\mu} J^{\mu}\right)+2 \imath q_{L} \int C\left(\bar{\psi}_{L} \frac{\delta_{l} \bar{S}}{\delta \bar{\psi}_{L}}-\frac{\delta_{r} \bar{S}}{\delta \psi_{L}} \psi_{L}\right),
\end{aligned}
$$

where $J^{\mu}=q_{L} \bar{\psi}_{L} \gamma^{\mu} \psi_{L}$ is the gauge current and $\bar{S}(\Phi)=$ $S(\Phi, 0)$.

We focus on the matter-independent contributions $\mathcal{A}_{B}$ to the anomaly $\mathcal{A}=\left\langle\left(S_{r 0}, S_{r 0}\right)\right\rangle_{S_{r 0}}$, so we can take the ghosts outside the average. Switching to momentum space, we get

$$
\begin{aligned}
\mathcal{A}_{B}= & -2 \imath q_{L} \int \frac{\mathrm{d}^{D} p}{(2 \pi)^{D}} C(-k) \\
& \times \operatorname{tr}\left[\hat{p}\left(P_{R}-P_{L}\right)\left\langle\psi\left(p+k_{1}\right) \bar{\psi}\left(-p+k_{2}\right)\right\rangle\right] .
\end{aligned}
$$

Here and below the integrals on momenta $k$ in $\mathcal{A}_{B}$ are understood. We expand the fermion two-point function in powers of the gauge field. The linear term gives a contribution that by power counting and ghost number conservation is proportional to

$\int(\square C)\left(\partial^{\mu} A_{\mu}\right)=\left(S_{K}, \chi^{\prime}\right), \quad \chi^{\prime}=\frac{1}{2} \int\left(\partial_{\mu} A_{\nu}\right)\left(\partial^{\mu} A^{\nu}\right)$.

It can be subtracted away as explained in (5.12). Then we concentrate on the contributions $\mathcal{A}_{B}^{\prime}$ to $\mathcal{A}_{B}$ that are quadratic in the gauge field. We observe that one fermion propagator is sandwiched between two $P_{L}$ 's or two $P_{R}$ 's, which projects its numerator onto the evanescent sector, and the other two propagators are sandwiched between $P_{L}$ and $P_{R}$, which projects their numerators onto the physical sector. We get

$$
\begin{aligned}
\mathcal{A}_{B}^{\prime}= & -2 q_{L}^{3} \int \frac{\mathrm{d}^{D} p}{(2 \pi)^{D}} \frac{C(-k) \hat{p}^{2}}{\left(p+k_{1}\right)^{2} p^{2}\left(p-k_{2}\right)^{2}} \\
& \times \operatorname{tr}\left[P_{L} \not \not A\left(k_{1}\right) \not \bar{p} A\left(k_{2}\right)\right] .
\end{aligned}
$$

The photons and their momenta $k_{1}, k_{2}$ can be taken to be strictly four dimensional. Turning to Euclidean space and using 


$$
\begin{aligned}
I^{\mu} & =\int_{E u c l} \frac{\mathrm{d}^{D} p}{(2 \pi)^{D}} \frac{\hat{p}^{2} \bar{p}^{\mu}}{\left(p+k_{1}\right)^{2} p^{2}\left(p-k_{2}\right)^{2}} \\
& =\frac{1}{96 \pi^{2}}\left(k_{1}^{\mu}-k_{2}^{\mu}\right)+\mathcal{O}(\varepsilon),
\end{aligned}
$$

we obtain

$\mathcal{A}_{B}^{\prime}=-\frac{q_{L}^{3}}{12 \pi^{2}} \int C(-k) \varepsilon^{\mu \nu \rho \sigma} k_{1 \mu} A_{\nu}\left(k_{1}\right) k_{2 \rho} A_{\sigma}\left(k_{2}\right)$,

where $\varepsilon^{0123}=1$. Converting to coordinate space and including the trivial contributions, we finally get

$\mathcal{A}_{B}=\frac{q_{L}^{3}}{48 \pi^{2}} \int C \varepsilon^{\mu \nu \rho \sigma} F_{\mu \nu} F_{\rho \sigma}+\left(S_{K}, \chi\right)$.

After subtraction of the trivial terms the divergence of the current averages to

$\left\langle\partial_{\mu} J^{\mu}\right\rangle=\frac{q_{L}^{3}}{96 \pi^{2}} \varepsilon^{\mu \nu \rho \sigma} F_{\mu \nu} F_{\rho \sigma}-\imath q_{L}\left(\bar{\psi}_{L} \frac{\delta_{l} \bar{S}}{\delta \bar{\psi}_{L}}-\frac{\delta_{r} \bar{S}}{\delta \psi_{L}} \psi_{L}\right)$.

Incidentally, the calculation shows that $\mathcal{A}_{B}$ receives no contributions proportional to $\int C F^{\mu \nu} F_{\mu \nu}$. This term is in principle allowed by the cohomological constraint (5.6) in Abelian theories, but actually does not show up. If it did, it would imply that the global symmetry associated with the gauge symmetry is anomalous, which is of course not true.

The calculation just done also proves (5.11), after inserting matrices $T^{a}$ and structure constants $f^{a b c}$ where appropriate.

\section{Appendix B: Formula of the anomaly functional}

In this appendix we recall the proof of the last equalities of (2.11) and (3.10), which express the anomaly functional $\mathcal{A}$. We show that

$(\Gamma, \Gamma)=\langle(S, S)\rangle$,

where $S$ is a dimensionally regularized action, the average is defined by the functional integral (2.10) and $\Gamma$ is the Legendre transform of $W$, defined by the same integral.

Recall that, using the dimensional regularization technique, local perturbative field redefinitions have Jacobian determinants identically equal to one. Indeed, from the diagrammatic point of view such Jacobians are equal to 1 plus integrals of polynomials of the momenta $p$ in $\mathrm{d}^{D} p$, which vanish. Now, if we make the change of field variables

$\Phi^{\alpha} \rightarrow \Phi^{\alpha}+\theta\left(S, \Phi^{\alpha}\right)=\Phi^{\alpha}-\theta \frac{\delta_{r} S}{\delta K_{\alpha}}$

in the functional integral (2.10), where $\theta$ is a constant anticommuting parameter, we obtain

$-\iota \theta \int\left\langle\frac{\delta_{r} S}{\delta K_{\alpha}} \frac{\delta_{l} S}{\delta \Phi^{\alpha}}\right\rangle-\iota \theta \int\left\langle\frac{\delta_{r} S}{\delta K_{\alpha}}\right\rangle J_{\alpha}=0$.
Using this identity, and recalling that the two terms of the antiparentheses (2.2) are equal when $X$ and $Y$ coincide and have bosonic statistics, we get

$\frac{1}{2}\langle(S, S)\rangle=-\int\left\langle\frac{\delta_{r} S}{\delta K_{\alpha}} \frac{\delta_{l} S}{\delta \Phi^{\alpha}}\right\rangle=\int\left\langle\frac{\delta_{r} S}{\delta K_{\alpha}}\right\rangle J_{\alpha}$.

The average of $\delta_{r} S / \delta K_{\alpha}$ is equal to $\delta_{r} W / \delta K_{\alpha}$, which is also $\delta_{r} \Gamma / \delta K_{\alpha}$, because the sources $K$ are inert in the Legendre transform that defines $\Gamma$. Using $J_{\alpha}=-\delta_{l} \Gamma / \delta \Phi^{\alpha}$, we arrive at

$\frac{1}{2}\langle(S, S)\rangle=\int \frac{\delta_{r} W}{\delta K_{\alpha}} J_{\alpha}=-\int \frac{\delta_{r} \Gamma}{\delta K_{\alpha}} \frac{\delta_{l} \Gamma}{\delta \Phi^{\alpha}}=\frac{1}{2}(\Gamma, \Gamma)$.

For other details, see for example the appendix of Ref. [19]. Note that the dimensional regularization is crucial for the derivation. Clearly, formula (B.1) also works if we use the DHD regularization, because the dimensional one is embedded in it. We then obtain (3.10).

If a dimensionally regularized action $S$ satisfies $(S, S)=0$ in arbitrary $D=4-\varepsilon$ dimensions, then gauge anomalies are manifestly absent, as in QED and QCD, and (2.11) correctly gives $\mathcal{A}=0$. When chiral fermions are present, as in the standard model, we have the $\gamma_{5}$ problem. A dimensionally regularized action $S$ cannot equip chiral fermions with wellbehaved propagators, and satisfy $(S, S)=0$ in $D$ dimensions at the same time. The naïve fermionic propagators, given by the starting action (2.1), do not depend on the evanescent components $\hat{p}$ of momenta. Then, according to the rules of the dimensional regularization, fermion loops integrate to zero, which means that the starting action (2.1) is not well regularized. The action must be modified to equip fermions with well-behaved propagators, for example by adding the correction $S_{\mathrm{LR}}$ of (2.6). Once this is done, however, $S$ satisfies $(S, S)=\mathcal{O}(\varepsilon)$, as shown in (2.9) and (3.5). The evanescent terms $\mathcal{O}(\varepsilon)$, inserted in the diagrams belonging to the average $\langle(S, S)\rangle=\langle\mathcal{O}(\varepsilon)\rangle$, can simplify poles $1 / \varepsilon$ and give finite, potentially anomalous contributions, as shown in the calculation of the previous appendix.

It is worth to stress that our investigation only concerns gauge anomalies, so $\mathcal{A}=0$ does not exclude the presence of other types of anomalies, such as the axial anomaly of QED.

\section{References}

1. S.L. Adler, W.A. Bardeen, Absence of higher order corrections in the anomalous axial vector divergence. Phys. Rev. 182, 1517 (1969)

2. S.L. Adler, Anomalies to all orders, in Fifty Years of Yang-Mills Theory, ed. by G. 't Hooft. (World Scientific, Singapore, 2005), pp. 187-228. arXiv:hep-th/0405040

3. S. Adler, Axial-vector vertex in spinor electrodynamics. Phys. Rev. 177, 2426 (1969)

4. J.S. Bell, R. Jackiw, A PCAC puzzle: $\pi^{0} \rightarrow \gamma \gamma$ in the $\sigma$-model. Nuovo Cim. A 60, 47 (1969)

5. A. Zee, Axial-vector anomalies and the scaling property of field theory. Phys. Rev. Letters 29, 1198 (1972) 
6. J. Collins, Renormalization, Chapter 13. (Cambridge University Press, Cambridge, 1984)

7. T. Marinucci, M. Tonin, Dimensional regularization and anomalies. Il Nuovo Cimento A 31, 381 (1976)

8. C. Lucchesi, O. Piguet, K. Sibold, The Adler-Bardeen theorem for the axial $\mathrm{U}(1)$ anomaly in a general non-Abelian gauge theory. Int. J. Mod. Phys. A 2, 385 (1987)

9. E. Witten, Global aspects of current algebra. Nucl. Phys. B 223, 422 (1983)

10. J. Wess, B. Zumino, Consequences of anomalous Ward identities. Phys. Lett. B 37, 95 (1971)

11. G. Costa, J. Julve, T. Marinucci, M. Tonin, Non-Abelian gauge theories and triangle anomalies. Nuovo Cimento A 38, 373 (1977)

12. O. Piguet, S. Sorella, Adler-Bardeen theorem and vanishing of the gauge beta function, Nucl. Phys. B 395661 (1993). arXiv:hep-th/9302123

13. D. Anselmi, Weighted power counting, neutrino masses and Lorentz violating extensions of the standard model, Phys. Rev.
D 7925017 (2009). 08A4 Renormalization.com. arXiv:0808.3475 [hep-ph]

14. D. Anselmi, M. Halat, Renormalization of Lorentz violating theories, Phys. Rev. D 76125011 (2007). arXiv:0707.2480 [hep-th]

15. I.A. Batalin, G.A. Vilkovisky, Gauge algebra and quantization. Phys. Lett. B 102, 27-31 (1981)

16. I.A. Batalin, G.A. Vilkovisky, Quantization of gauge theories with linearly dependent generators, Phys. Rev. D 282567 (1983). Erratum-ibid. D 30508 (1984)

17. S. Weinberg, The Quantum Theory of Fields, vol. II. (Cambridge University Press, Cambridge, 1995)

18. W. Bardeen, Anomalous Ward identities in spinor field theories. Phys. Rev. 184, 1848 (1969)

19. D. Anselmi, Background field method, Batalin-Vilkovisky formalism and parametric completeness of renormalization. Phys. Rev. D 89045004 (2014). 13A3 Renormalization.com. arXiv:1311.2704 [hep-th] 